\title{
Non-Expected Utility and the Robustness of the Classical Insurance Paradigm
}

MARK J. MACHINA

Department of Economics, University of California-San Diego, La Jolla, California 92093-0508, U.S.A.

\begin{abstract}
This paper uses the tools and techniques of generalized expected utility analysis to explore the robustness of some of the classical basic results in insurance theory to departures from the expected utility hypothesis on agents' risk preferences. The areas explored consist of individual demand for coinsurance and deductible insurance, the structure of Pareto-efficient bilateral insurance contracts, the structure of Pareto-efficient multilateral risk-sharing agreements, and self-insurance and self-protection. Most, though not all, of the basic results in this area are found to be quite robust to dropping the expected utility hypothesis.
\end{abstract}

Key words: insurance, risk sharing, non-expected utility

\section{Introduction}

The purpose of this paper is to explore what the fields of non-expected utility theory and the economic theory of insurance might have to contribute to each other.

For the benefit of readers more familiar with insurance theory than with non-expected utility, I begin by describing what non-expected utility risk preferences are, and the different ways-both algebraic and graphical-to study them. The first point I'm going to make is that non-expected utility is not an alternative to expected utility. Rather, it is a generalization of it, much like CES utility functions over commodity bundles are generalizations of Cobb-Douglas utility functions or, better still, like general quasiconcave functions are generalizations of Cobb-Douglas functions.

Accordingly, I would like to ask the reader to think of the theory of insurance as developed under the expected utility hypothesis as similar to the situation of someone who has developed the theory of consumer demand using only Cobb-Douglas utility functions. Such a Cobb-Douglas scientist has an easy and tractable model to work with, and he or she is likely to discover and derive many results, such as the Slutsky equation, or that income elasticities are identically one, or that cross-price elasticities are identically zero. But we know that while the Slutsky equation is a general property of all utility functions over commodity bundles, the two elasticity results are specific to the Cobb-Douglas case and most definitely not true of more general utility functions. It is had to see how our scientist could have known the robust results from the nonrobust results, unless he or she at least took a peek at more general non-Cobb-Douglas preferences. 
The goal of this paper is to examine some of the classic theoretical results in individual and market insurance theory from the more general non-expected utility point of view and determine which of these classic results are robust (like the Slutsky equation) and which are not. I mentioned that I am interested in what non-expected utility theory and insurance theory can contribute to each other. The identification of the robust results can contribute to insurance theory, by determining which theorems can be "leaned on" most heavily for further theoretical implications. The identification of the nonrobust results can contribute to non-expected utility theory by determining which parts of current insurance theory are in effect testable implications of the expected utility hypothesis. Since insurance provides the largest, most systematic, and most intensive set of field data on both individual and market choices under uncertainty, this would provide us non-expected utility types with a desperately needed opportunity to apply real-world data to the testing of the expected utility model and the calibration of our more general models of choice under uncertainty.

Experts in insurance theory will note that the selection of results I have chosen to examine reflects breadth rather than depth. This reflects my own less-than-exhaustive knowledge of the huge literature on insurance. It also reflects the fact that more specific and sophisticated results often require more specialized assumptions (such as convexity of marginal utility or HARA utility functions), whose natural generalizations to non-expected utility may have yet to be worked out. But I feel that we can learn most about robustness by starting out with an examination of the most basic and fundamental results in each of the various branches of insurance theory.

Section 2 of this paper introduces the notion of non-expected utility preferences over lotteries and describes how they are represented and analyzed, both graphically and algebraically. The next several sections use these tools to examine the robustness of the classic results of insurance theory to these more general types of risk preferences. Section 3 covers the individual's demand for insurance, taking the form of the insurance contract (coinsurance or deductible) as given. Section 4 examines the determination of the optimal form of insurance contract. Section 5 considers the general conditions for Pareto-efficient risk sharing among many individuals. Finally, Section 6 examines the classic problem of selfinsurance versus self-protection. I conclude with some thoughts on further work.

\section{Non-expected utility preferences and non-expected utility analysis}

Non-expected utility theory-at least the way I look at it-works with the same objects of choice as standard insurance theory-namely, lotteries over final wealth levels, which can be represented by discrete probability distributions of the form $\mathbf{P}=\left(x_{1}, p_{1} ; \ldots ; x_{n}, p_{n}\right)$ or, in more general analyses, by cumulative distribution functions $F(\cdot) .{ }^{1}$ Non-expected utility theory also follows the standard approach by assuming-or positing axioms sufficient to imply-that the individual's preferences over such lotteries can be represented by means of a preference function $V(P)=V\left(x_{1}, p_{1} ; \ldots ; x_{n}, p_{n}\right)$. Just as with preferences over commodity bundles, the preference function $V(\cdot)$ can be analyzed graphically, by means of its indifference curves, or algebraically.

In our discussion of non-expected utility preferences, it will be useful to keep in mind the benchmark special case of expected utility. Recall that under the expected utility hypothesis, $\nabla(\cdot)$ takes the specific form 


$$
\vee\left(x_{1}, p_{1} ; \ldots ; x_{n}, p_{n}\right) \equiv \sum_{i=1}^{n} U\left(x_{i}\right) \cdot p_{i}
$$

for some von Neumann-Morgenstern utility function $U(\cdot)$.

Now, the normative appeal of the expected utility axioms is well known. However, in their capacity as descriptive economists, non-expected utility theorists wonder whether restricting attention solely to the functional form (1) might not be like the Cobb-Douglas hypothesis of our above scientist. We would like to determine which results of classic risk and insurance theory follow because of that functional form and which might follow from the properties of risk aversion or stochastic dominance preference in general, without really requiring the functional form (1). To do this, we begin by illustrating how non-expected utility theorists analyze general preference functions $\nabla\left(x_{1}, p_{1} ; \ldots ; x_{n}, p_{n}\right)$ and how they compare them to expected utility.

\subsection{Graphical depictions of non-expected utility preferences}

As might be expected, some simple diagrams can help illustrate the key differences between expected utility preferences and non-expected utility preferences, by depicting how preferences over distributions $P=\left(x_{1}, p_{1} ; \ldots ; x_{n}, p_{n}\right)$ depend on: (1) changes in the outcomes $\left\{x_{1}, \ldots, x_{n}\right\}$ for a fixed set of probabilities $\left\{\bar{p}_{1}, \ldots, \bar{p}_{n}\right\}$; and (2) changes in the probabilities $\left\{p_{1}, \ldots, p_{n}\right\}$ for a fixed set of outcomes $\left\{\bar{x}_{1}, \ldots, \bar{x}_{n}\right\}$.

Preferences over changes in the outcomes can be illustrated in the classic HirshleiferYaari diagram (Hirshleifer [1965, 1966], Yaari [1965, 1969], Hirshleifer and Riley [1979, 1992]). Assume there are two states of nature, with fixed, known probabilities $\left(\bar{p}_{1}, \bar{p}_{2}\right)$ adding to unity, so that we restrict attention to probability distributions of the form $\left(x_{1}, \bar{p}_{1}\right.$; $\left.x_{2}, \bar{p}_{2}\right)$, which can be represented by points in the $\left(x_{1}, x_{2}\right)$ plane, as in Figure 1. A family of expected utility indifference curves in this diagram are the level curves of some expected utility preference function $\vartheta(\mathbf{P})=U\left(x_{1}\right) \cdot \bar{p}_{1}+U\left(x_{2}\right) \cdot \bar{p}_{2}$, with slope (marginal rate of substitution) naturally given by

$$
\operatorname{MRS}_{E U}\left(x_{1}, x_{2}\right) \equiv-\frac{U^{\prime}\left(x_{1}\right) \cdot \bar{p}_{1}}{U^{\prime}\left(x_{2}\right) \cdot \bar{p}_{2}}
$$

Besides indifference curves, Figure 1 also contains two other constructs. The $45^{\circ}$ line consists of all sure prospects $(x, x)$ and is accordingly termed the certainty line. The parallel dashed lines are loci of constant expected value $x_{1} \cdot \bar{p}_{1}+x_{2} \cdot \bar{p}_{2}$, with slope accordingly given by the odds ratio $-\bar{p}_{1} / \bar{p}_{2}$. In insurance theory these lines are frequently termed the fair odds lines, in non-expected utility theory, we call them iso-expected value lines.

Formula (2) implies two very specific properties of expected utility indifference curves in the Hirshleifer-Yaari diagram. It is straightforward to show that it implies both

- $M R S$ at certainty $=$ odds ratio. The $M R S$ at every point $(x, x)$ on the $45^{\circ}$ line equals the odds ratio $-\bar{p}_{1} / \bar{p}_{2}$, and

- Rectangle property. For any four points $\left(x_{1}^{*}, x_{2}^{*}\right),\left(x_{1}^{*}, x_{2}^{* *}\right),\left(x_{1}^{* *}, x_{2}^{*}\right),\left(x_{1}^{* *}, x_{2}^{* *}\right)$ that form a rectangle in the diagram, the products of the $M R S$ 's at diagonally opposite pairs are equal. ${ }^{2}$ 


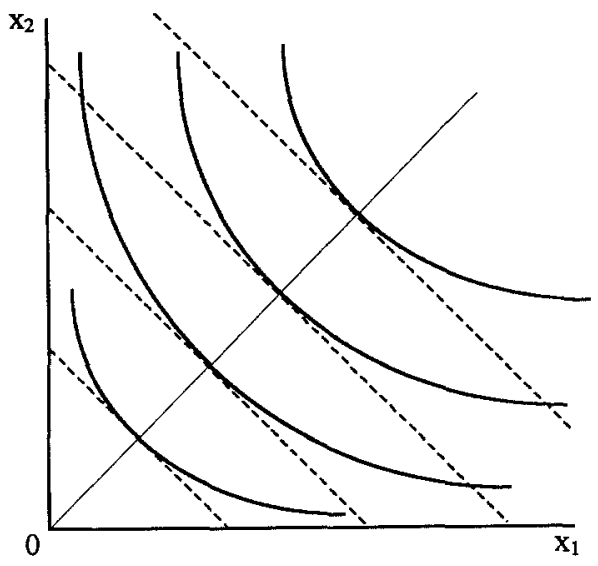

Figure 1. Risk averse expected utility indifference curves in the Hirshleifer-Yaari diagram.

Besides these two properties, the indifference curves drawn in Figure 1 exhibit three other features of risk preferences on the part of the underlying preference function $\nabla(\cdot)$ that generates them. The first feature is that they are downward sloping. To see what this reflects, note that any north, east, or northeast movement in the diagram will, by raising $x_{1}$ or $x_{2}$, lead to a (first-order) stochastically dominating probability distribution. Accordingly, any set of indifference curves that is downward sloping is reflecting stochastic dominance preference on the part of its underyling preference function $\nabla(\cdot)$. Of course, under expected utility, this is equivalent to the condition that $U\left({ }^{\circ}\right)$ is an increasing function of $x$.

The second feature of these indifference curves is that they are steeper than the iso-expected value lines in the region above the $45^{\circ}$ line and flatter than the iso-expected value curves in the region below the $45^{\circ}$ line. To see what this reflects, note that, starting at any point $\left(x_{1}, x_{2}\right)$ and moving along its iso-expected value line in a direction away from the certainty line serves to further increase the larger outcome of the probability distribution and further decrease the smaller outcome, and does so in a manner that preserves the expected value of the prospect. This is precisely a mean preserving increase in risk. ${ }^{3}$ Thus, indifference curves that are steeper or flatter than the iso-expected values lines in the region above or below the certainty line are made worse off by all such increases in risk and hence reflect the property of risk aversion on the part of their underlying preference function $\checkmark(\cdot)$. Under expected utility, this property is equivalent to the condition that $U(\cdot)$ is a concave function of $x$.

The third feature of the indifference curves in Figure 1 is that they are bowed-in toward the origin. This means that a convex combination $\left(\lambda \cdot x_{1}+(1-\lambda) \cdot x_{1}^{*}, \lambda \cdot x_{2}+(1-\lambda)\right.$ - $\left.x_{2}^{*}\right)$ of any two indifferent points $\left(x_{1}, x_{2}\right)$ and $\left(x_{1}^{*}, x_{2}^{*}\right)$ will be preferred to these points. Expressed more generally, we term this property outcome convexity-namely, for any set of probabilities $\left\{\bar{p}_{1}, \ldots, \bar{p}_{n}\right\}$,

$$
\begin{aligned}
& \left(x_{1}, \bar{p}_{1} ; \ldots ; x_{n}, \bar{p}_{n}\right) \sim\left(x_{1}^{*}, \bar{p}_{1} ; \ldots ; x_{n}^{*} \bar{p}_{n}\right) \Rightarrow \\
& \quad\left(\lambda \cdot x_{1}+(1-\lambda) \cdot x_{1}^{*}, \bar{p}_{1} ; \ldots ; \lambda \cdot x_{n}+(1-\lambda) \cdot x_{n}^{*}, \bar{p}_{n}\right) \succeq\left(x_{1}, \bar{p}_{1} ; \ldots ; x_{n}, \bar{p}_{n}\right)
\end{aligned}
$$


for all $\lambda \in(0,1)$. This property of risk preferences has been examined, under various names, by Tobin [1958], Debreu [1959, ch. 7], Yaari [1965, 1969], Dekel [1989], and Karni [1992]. Under expected utility, it is equivalent to the condition that $U(\cdot)$ is concave.

Note what these last two paragraphs imply: Since under expected utility the properties of risk aversion and outcome convexity are both equivalent to concavity of $U(\cdot)$, it follows that expected utility indifference curves in the plane-and expected utility preferences in general-will be risk averse if and only if they are outcome convex. We will see the implications of this below.

A family of non-expected utility indifference curves, on the other hand, consists of the level curves of some general preference function $\mathcal{V}(\mathbf{P})=\mathcal{V}\left(x_{1}, \bar{p}_{1} ; x_{2}, \bar{p}_{2}\right)$, with slope therefore given by

$$
\operatorname{MRS}_{\curlyvee}\left(x_{1}, x_{2}\right) \equiv-\frac{\partial \mathcal{V}\left(x_{1}, \bar{p}_{1} ; x_{2}, \bar{p}_{2}\right) / \partial x_{1}}{\partial \bigvee\left(x_{1}, \bar{p}_{1} ; x_{2}, \bar{p}_{2}\right) / \partial x_{2}}
$$

Two such examples, derived from two different preference functions $\nabla_{a}\left({ }^{\circ}\right)$ and $\nabla_{b}(\cdot)$, are illustrated in Figure 2. Keep in mind that in Figures 2a and 2b, just as in Figure 1, the indifference curves are generated by an underlying preference function $V(\cdot)$ defined over the probability distributions implied by each $\left(x_{1}, x_{2}\right)$ pair under the well-defined state probabilities $\left(\bar{p}_{1}, \bar{p}_{2}\right)$ : we refer to such preferences over $\left(x_{1}, x_{2}\right)$ bundles as probabilistically sophisticated.

Expected utility and non-expected utility preference functions, and hence their respective indifference maps, have two features in common and two important differences. Their first common feature is stochastic dominance preference. Stochastic dominance preference is the stochastic analogue of "more money is better" and makes just as much sense under non-expected utility as under expected utility. As we have seen, this translates into downward sloping indifference curves in the Hirshleifer-Yaari diagram and is reflected in both parts of Figure 2.

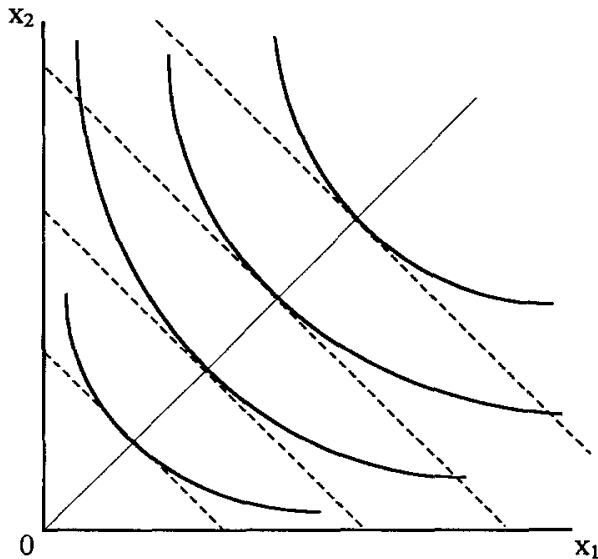

(a)

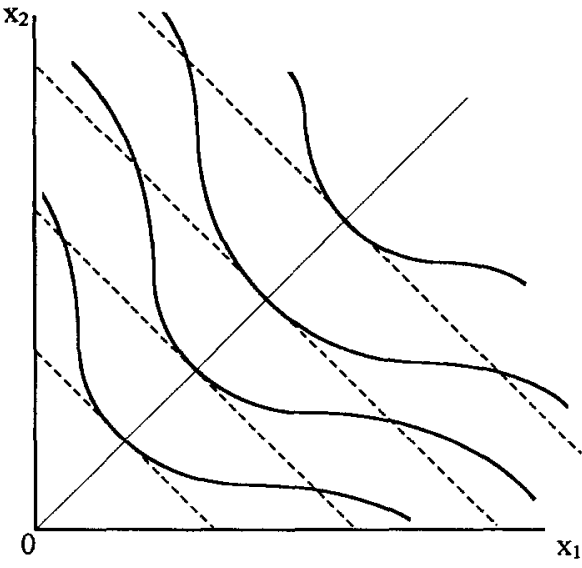

(b)

Figure 2. Risk-averse non-expected utility indifference curves. (a) Outcome convex. (b) Non-outcome convex. 
The second common feature is the "MRS at certainty = odds ratio" condition, as seen in both parts of Figure 2. The non-expected utility condition for this property-namely, that any sufficiently smooth non-expected utility preference function $\vee\left({ }^{\circ}\right)$ must satisfy

$$
\operatorname{MRS}_{\vartheta}(x, x) \equiv-\left.\frac{\partial \vartheta\left(x_{1}, \bar{p}_{1} ; x_{2}, \bar{p}_{2}\right) / \partial x_{1}}{\partial \nabla\left(x_{1}, \bar{p}_{1} ; x_{2}, \bar{p}_{2}\right) / \partial x_{2}}\right|_{x_{1}=x_{2}=x}=-\frac{\bar{p}_{1}}{\bar{p}_{2}}
$$

follows from an early result of Samuelson [1960, pp. 34-37, eq. 5]. Note that it implies that we can recover a non-expected utility (or expected utility) maximizer's subjective probabilities from their indifference curves over state-indexed outcomes in the Hirshleifer-Yaari diagram.

The first of the two important differences between expected utility and non-expected utility should not come as a surprise. Any departure from the additively separable expected utility form (1) means that the so-called rectangle property on MRS's will no longer hold.4 This is a well-known consequence for indifference curves over any kind of commodities, once we drop the assumption of separability of the preference function that generates them.

We come now to the second important difference between expected utility and nonexpected utility indifference curves-one that will play a very important role in our analysis. Note that while the non-expected utility indifference curves of Figure 2a needn't satisfy the rectangle property for $M R S$ 's, they do satisfy both risk aversion ${ }^{5}$ and outcome convexity-just like the expected utility indifference curves of Figure 1. However, the non-expected utility indifference curves of Figure $2 \mathrm{~b}$ are risk averse but not outcome convex. In other words, in the absence of the expected utility hypothesis, risk aversion is no longer equivalent to outcome convexity, and as Dekel [1989] has formally shown, it is quite possible for a preference function $\nabla(\cdot)$, and hence its indifference curves, to be globally risk averse but not outcome convex. ${ }^{6}$

On the other hand, Dekel has shown that if a non-expected utility $\nabla(\cdot)$ is outcome convex then it must be risk averse. Although this is a formal result that applies to preferences over general probability distributions, the graphical intuition can be seen from Figure 2a: recall that non-expected utility indifference curves must be tangent to the iso-expected value lines. Thus, if they are also outcome convex, they must be steeper than these lines above the $45^{\circ}$ line and flatter than those below the $45^{\circ}$ line, which is exactly the condition for risk aversion in the diagram.

Thus, in the absence of the expected utility hypothesis, risk aversion is seen to be a logically separate-and weaker-property than outcome convexity. This means that when dropping the expected utility hypothesis and examining the robustness of some insurance theorem that "only required risk aversion," we have to determine it really was "only risk aversion" that had been driving the result in question or whether it was risk aversion plus outcome convexity that had been doing so.

Let us now illustrate preferences over change in the probabilities for fixed outcome values. Specifically, pick any three values $\bar{x}_{1}<\bar{x}_{2}<\bar{x}_{3}$, and consider the set of all probability distributions of the form $\left(\bar{x}_{1}, p_{1} ; \bar{x}_{2}, p_{2} ; \bar{x}_{3}, p_{3}\right)$. Since we must have $p_{2}=1-p_{1}-p_{3}$, we can plot each of these distributions as a point $\left(p_{1}, p_{3}\right)$ plane, as in Figure 3. Once again, a family of expected utility indifference curves will consist of the level curves of some 


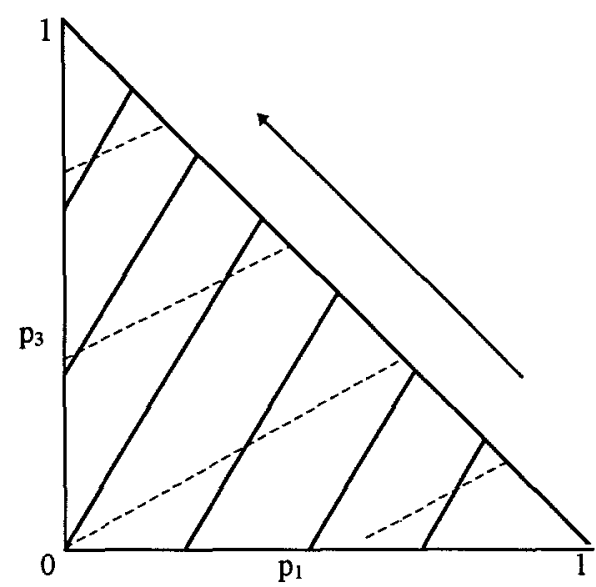

(a)

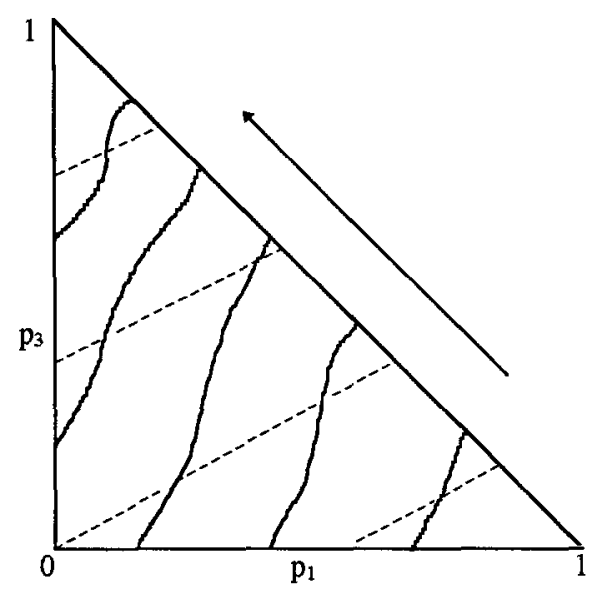

(b)

Figure 3. Risk averse expected utility (a) and non-expected utility (b) indifference curves in the probability triangle.

expected utility preference functional $V(\mathbf{P})=U\left(\bar{x}_{1}\right) \cdot p_{1}+U\left(\bar{x}_{2}\right) \cdot p_{2}+U\left(\bar{x}_{3}\right) \cdot p_{3}$, which, after substituting for $p_{2}$, takes the form

$$
U\left(\bar{x}_{2}\right)+\left[U\left(\bar{x}_{3}\right)-U\left(\bar{x}_{2}\right)\right] \cdot p_{3}-\left[U\left(\bar{x}_{2}\right)-U\left(\bar{x}_{1}\right)\right] \cdot p_{1}
$$

with $M R S$ accordingly given by

$$
M R S_{E U}\left(p_{1}, p_{3}\right) \equiv \frac{U\left(\bar{x}_{2}\right)-U\left(\bar{x}_{1}\right)}{U\left(\bar{x}_{3}\right)-U\left(\bar{x}_{2}\right)}
$$

A family of non-expected utility indifference curves in the $\left(p_{1}, p_{3}\right)$ diagram consist of the level curves of some general preference function $\nabla\left(\bar{x}_{1}, p_{1} ; \bar{x}_{2}, p_{2} ; \bar{x}_{3}, p_{3}\right)$, subject to $p_{2}=1-p_{1}-p_{3}$. Substituting for $p_{2}$ to obtain the expression $\nabla\left(\bar{x}_{1}, p_{1} ; \bar{x}_{2}, 1-p_{1}-p_{3}\right.$; $\left.\bar{x}_{3}, p_{3}\right)$, we have that the slope of these indifference curves at any point $\left(p_{1}, p_{3}\right)$ is given by the formula

$$
\left.M R S_{\vee}\left(p_{1}, p_{3}\right) \equiv \frac{\frac{\partial \mathcal{V}(\mathbf{P})}{\partial p_{2}}-\frac{\partial \mathcal{V}(\mathbf{P})}{\partial p_{1}}}{\frac{\partial \mathcal{P}(\mathbf{P})}{\partial p_{3}}-\frac{\partial \mathscr{P}(\mathbf{P})}{\partial p_{2}}}\right|_{\mathbf{P}=\left(\bar{x}_{1}, p_{1} ; \bar{x}_{2}, 1-p_{1}-p_{3} ; \bar{x}_{3}, p_{3}\right)}
$$

Figure 3a highlights the single most significant feature of expected utility preferencesnamely, the property of linearity in the probabilities. As the level curves of a linear function (formula (1) or (6)), expected utility indifference curves in the probability diagram are parallel straight lines. This is the source of much of the predictive power of the expected utility model, since it implies that knowledge of indifference curves in any one region of the triangle implies knowledge of them over the whole triangle. 
As we did for the Hirshleifer-Yaari diagram, we can ask what the properties of stochastic dominance preference and risk aversion look like in the probability triangle. A pure northward movement in the triangle implies a rise in $p_{3}$ along with, of course, a matching drop in $p_{2}$. This corresponds to shifting probability from the outcome $\bar{x}_{2}$ up to the higher outcome $\bar{x}_{3}$. A westward movement implies a drop in $p_{1}$ with matching rise in $p_{2}$. An exact $\left(45^{\circ}\right)$ northwestward movement implies a rise in $p_{3}$ with equal drop in $p_{1}$ (no change in $p_{2}$ ). All three of these movements shift probability mass from some lower outcome up to some higher outcome and hence are stochastically dominating shifts. Since the sets of indifference curves in both parts of Figure 3 are both upward sloping, they prefer such shifts and hence both reflect stochastic dominance preference.

The property of risk aversion is once again illustrated by reference to iso-expected value lines. In the probability triangle, they are the (dashed) level curves of the formula

$\bar{x}_{1} \cdot p_{1}+\bar{x}_{2} \cdot\left(1-p_{1}-p_{3}\right)+\bar{x}_{3} \cdot p_{3}=\bar{x}_{2}+\left[\bar{x}_{3}-\bar{x}_{2}\right] \cdot p_{3}-\left[\bar{x}_{2}-\bar{x}_{1}\right] \cdot p_{1}$

and hence have slope $\left[\bar{x}_{2}-\bar{x}_{1}\right] /\left[\bar{x}_{3}-\bar{x}_{2}\right]$. Northeast movements along these lines increase both of the outer (that is, the tail) probabilities $p_{1}$ and $p_{3}$ at the expense of the middle probability $p_{2}$, in a manner that does not change the expected value, so they represent the mean preserving spreads in the triangle. Since the indifference curves in both parts of Figure 3 are all steeper than these lines, they are made worse off by such increases in risk, and hence are both risk averse.

Besides risk aversion per se, these diagrams can also illustrate comparative risk aversionthe property that one individual is at least as risk averse as another. Arrow [1965b] and Pratt [1964] have shown that the algebraic condition for comparative risk aversion under expected utility is that a pair of utility function $U_{1}(\cdot)$ and $U_{2}(\cdot)$ satisfy the equivalent conditions

$$
\begin{array}{rlrl}
U_{1}(x) & \equiv \varphi\left(U_{2}(x)\right) & \text { for some increasing concave } \varphi(\cdot), \\
-\frac{U_{1}^{\prime \prime}(x)}{U_{1}^{\prime}(x)} \geq-\frac{U_{2}^{\prime \prime}(x)}{U_{2}^{\prime}(x)} & \text { for all } x, \\
\frac{U_{1}^{\prime}\left(x^{*}\right)}{U_{1}^{\prime}(x)} \leq \frac{U_{2}^{\prime}\left(x^{*}\right)}{U_{2}^{\prime}(x)} & \text { for all } x^{*}>x .
\end{array}
$$

Figure 4 illustrates the implications of these algebraic conditions for indifference curves in the Hirshleifer-Yaari and the triangle diagrams. The indifference curves of the more riskaverse utility function $U_{1}\left({ }^{\circ}\right)$ are solid; those of $U_{2}\left({ }^{\circ}\right)$ are dotted. In the Hirshleifer-Yaari diagram, the $M R S$ formula (2) and inequality (12) imply that the indifference curves of the more risk-averse $U_{1}\left({ }^{\circ}\right)$ are flatter than those of $U_{2}\left({ }^{\circ}\right)$ below the $45^{\circ}$ line and steeper than they are above it. In the triangle diagram, the $M R S$ formula (7) and a bit of calculus applied to either (11) or (12) yields that the indifference curves of the more risk-averse $U_{1}\left({ }^{\circ}\right)$ are steeper than those of $U_{2}\left({ }^{\circ}\right)$.

Comparing both parts of Figure 4 with Figures 1 and 3a reveals that in each case, the relative slope conditions for comparative risk aversion are simply a generalization of the slope conditions for risk aversion per se. This is such a natural result that we would want 


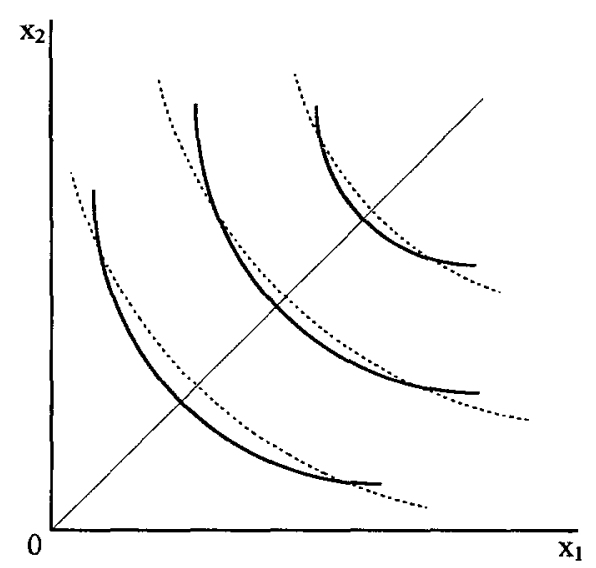

(a)

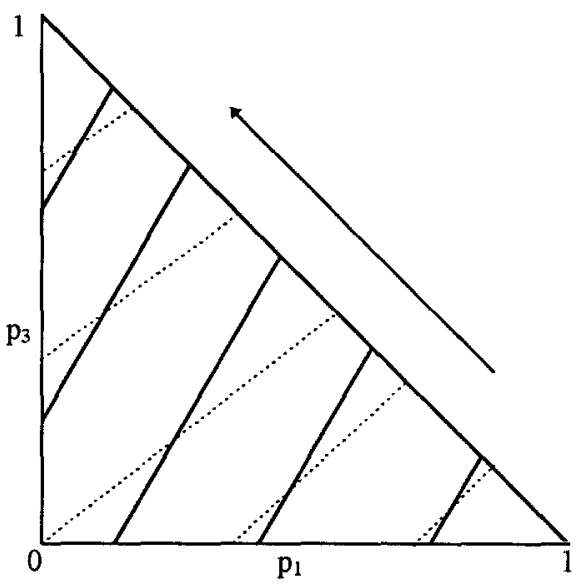

(b)

Figure 4. Comparative risk aversion for expected utility indifference curves.

to adopt it for non-expected utility indifference curves as well. In other words, when we come to determine the algebraic condition for comparative risk aversion under non-expected utility, we would insist that it imply these same relative slope conditions on indifference curves, as in Figure 5.

\subsection{Algebraic analysis of non-expected utility preferences}

What about algebraic analysis in the absence of expected utility? Let's go back and think about how we might reassure our Cobb-Douglas scientists, puzzled at having to drop their well-structured formula $c_{1}^{\alpha_{1}} \ldots \cdot c_{m}^{\alpha_{m}}$ for a shapeless general preference function $\mathcal{U}\left(c_{1}\right.$, $\left.\ldots, c_{m}\right)$. We would tell them that we conduct our general analysis in terms of the derivatives $\left\{\left(\partial \mathcal{U}(C) / \partial c_{1}\right), \ldots,\left(\partial \mathcal{U}(C) / \partial c_{n}\right)\right\}$ of our general function and that conditions on these derivatives, or maybe the ratios of these derivatives (marginal rates of substitution), give us theorems about behavior.

In fact, one branch ${ }^{7}$ of non-expected utility theory proceeds similarly, by working with derivatives of the preference function $\mathcal{V}(\cdot)$, and it is here that much of the robustness of expected utility analysis reveals itself. By way of motivation, let us recall some of the classical results of expected utility theory. For purposes of this exercise, assume that the set of potential outcome values $x_{1}<\ldots<x_{n}$ is fixed, so that only the probabilities $\left\{p_{1}, \ldots, p_{n}\right\}$ are independent variables. Now, given an expected utility preference function $\vee(P)=\Sigma_{i=1}^{n}$ $U\left(x_{i}\right) \cdot p_{i}$, don't think of $U\left(x_{i}\right)$ in its psychological role as the utility of outcome $x_{i}$ but rather in its purely mathematical role as the coefficient of $p_{i}=\operatorname{prob}\left(x_{i}\right)$. If we plot these probability coefficients against $x_{i}$, as in Figure $6 \mathrm{a}$, we can state the three most fundamental results of expected utility theory as follows:

- Stochastic dominance. $\nabla(\cdot)$ exhibits stochastic dominance preference if and only if its probability coefficients $\left\{U\left(x_{i}\right)\right\}$ form an increasing sequence, as in Figure 6 a. 


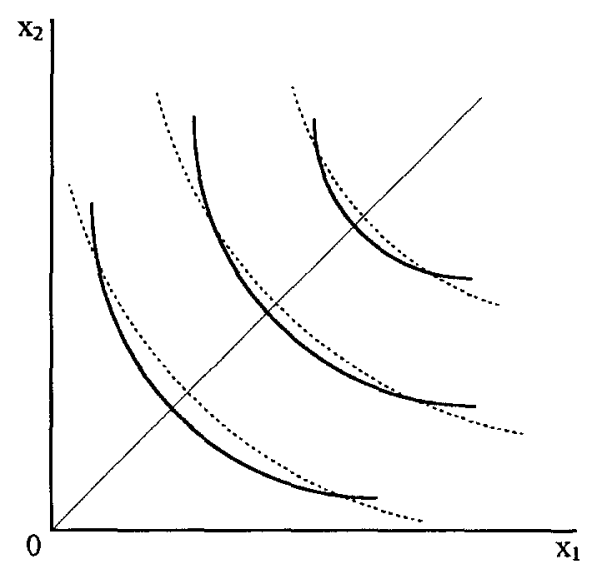

(a)

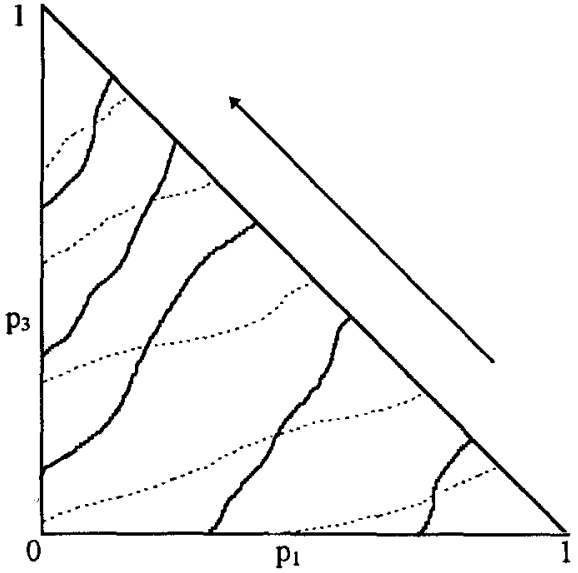

(b)

Figure 5. Comparative risk aversion for non-expected utility indifference curves.

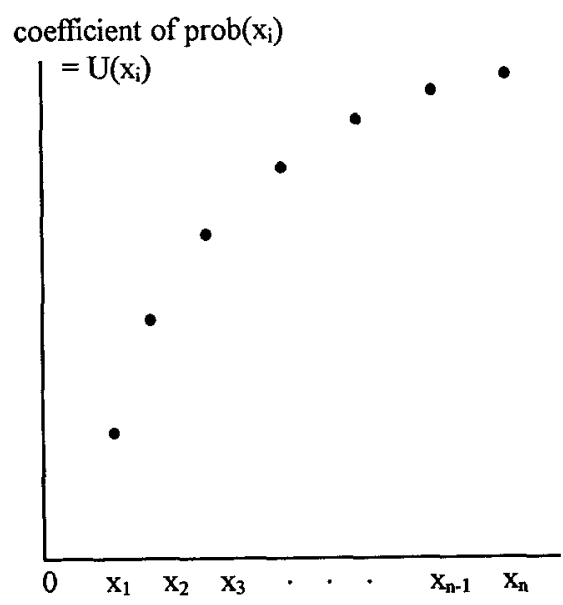

(a)

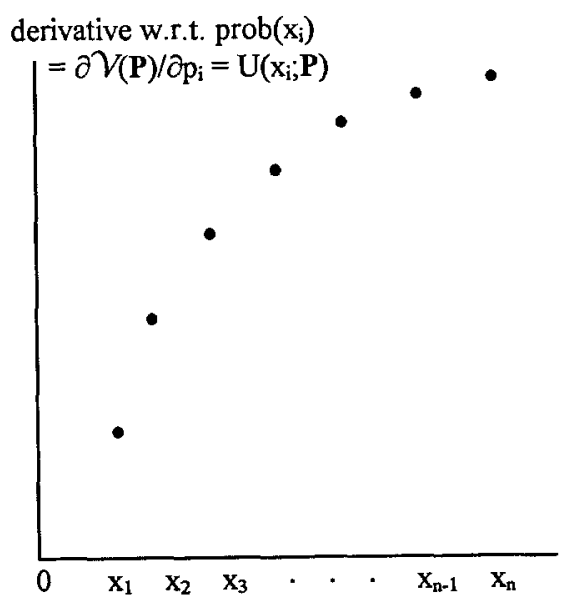

(b)

Figure 6. Expected utility probability coefficients (a) and non-expected utility probability derivatives (b) plotted against their corresponding outcome values.

- Risk aversion. $\checkmark(\cdot)$ is risk averse if and only if its probability coefficients $\left\{U\left(x_{i}\right)\right\}$ form a concave sequence, ${ }^{8}$ as in Figure 6a.

- Comparative risk aversion. $\nabla_{a}(\cdot)$ is at least as risk averse as $\nabla_{b}(\cdot)$ if and only if the sequence of probability coefficients $\left\{U_{a}\left(x_{i}\right)\right\}$ is at least as concave ${ }^{9}$ as the sequence of probability coefficients $\left\{U_{b}\left(x_{i}\right)\right\}$.

Now, consider a general non-expected utility preference function $\mathscr{V}(P)=\mathcal{V}\left(x_{1}, p_{1} ; \ldots\right.$; $\left.x_{n}, p_{n}\right)$, and continue to treat the outcomes $x_{1}<\ldots<x_{n}$ as fixed and the probabilities 
$\left\{p_{1}, \ldots, p_{n}\right\}$ as independent variables. Since $\nabla(\cdot)$ is not linear in the probabilities (not expected utility), it will not have probability coefficients. However, as long as $\nabla(\cdot)$ is differentiable, it will have a set of probability derivatives $\left\{\left(\partial \vee(P) / \partial p_{1}\right), \ldots,\left(\partial \vee(P) / \partial p_{n}\right)\right\}$ at each distribution $P$, and calculus tells us that in many cases, theorems based on the coefficients of a linear function will also apply to the derivatives of a nonlinear function.

Fortunately, this is precisely the case with the above three results, and this extension from probability coefficients to probability derivatives is the essence of generalized expected utility analysis. In other words, for any non-expected utility preference functional $\checkmark(\cdot)$, pick a distribution $P$, and plot the corresponding sequence of probability derivatives $\left\{\left(\partial \vee(\boldsymbol{P}) / \partial p_{1}\right), \ldots,\left(\partial \vee(\boldsymbol{P}) / \partial p_{n}\right)\right\}$ against $x_{i}$, as in Figure $6 \mathrm{~b}$. If these form an increasing sequence (as in the figure), then any infinitesimal stochastically dominating shift-say, an infinitesimal drop in $p_{i}$ and matching rise in $p_{i+1}$-will clearly be preferred. If the derivatives form a concave sequence (as in the figure), then any infinitesimal mean preserving increase in risk-such as an infinitesimal drop in $p_{i}$ coupled with a mean preserving rise in $p_{i-1}$ and $p_{i+1}$-will make the individual worse off.

Of course, these results are local, since they link the derivatives $\left\{\left(\partial \vee(P) / \partial p_{1}\right), \ldots\right.$, $\left.\left(\partial \mathcal{V}(P) / \partial p_{n}\right)\right\}$ at a distribution $P$ only to infinitesimal changes from $P$. However, we can take advantage of another feature of calculus-namely, that global conditions on derivatives are frequently equivalent to global properties of a function. Fortunately, this is the case with our three fundamental results. Thus, if the derivatives $\left\{\left(\partial \vee(P) / \partial p_{1}\right), \ldots,\left(\partial \vee(P) / \partial p_{n}\right)\right\}$ are found to form an increasing and concave sequence at all such distributions $P$, then $g l o b a l$ stochastically dominating shifts will always be preferred, and global increase in risk will always make the individual worse off, and so on. Formally, we can prove

- Stochastic dominance. A non-expected utility preference function $\nabla(\cdot)$ exhibits global stochastic dominance preference if and only if at each distribution $P$, its probability derivatives $\left\{\left(\partial \mathcal{V}(P) / \partial p_{i}\right)\right\}$ form an increasing sequence, as in Figure $6 \mathrm{~b}$.

- Risk aversion. $V\left({ }^{\circ}\right)$ is globally risk averse (averse to mean preserving increases in risk) if and only if at each $P$ its probability derivatives $\left\{\left(\partial \vee(P) / \partial p_{i}\right)\right\}$ form a concave sequence, as in Figure 6b.

- Comparative risk aversion. $\nabla_{a}(\cdot)$ is globally at least as risk averse $\operatorname{as}^{10} \nabla_{b}(\cdot)$ if and only if at each $P$, the sequence of probability derivatives $\left\{\left(\partial \nabla_{a}(P) / \partial p_{i}\right)\right\}$ is at least as concave as the sequence of probability derivatives $\left\{\left(\partial \nabla_{b}(P) / \partial p_{i}\right)\right\}$.

In light of this correspondence between expected utility's probability coefficients $\left\{U\left(x_{i}\right)\right\}$ and non-expected utility's probability derivatives $\left\{\left(\partial \vee(P) / \partial p_{i}\right)\right\}$, we adopt the suggestive notation $U\left(x_{i} ; P\right)=\left(\partial \widetilde{V}(P) / \partial p_{i}\right)$, and call $\left\{U\left(x_{i} ; P\right)\right\}$ the local utility index of ${ }^{\top}(\cdot)$ at $P$.

An important point: Do we really need to restrict ourselves just to changes in the probabilities of the original outcomes $\left\{x_{1}, \ldots, x_{n}\right\}$ ? No. At any distribution $P=\left(x_{1}, p_{1} ; \ldots\right.$; $\left.x_{n}, p_{n}\right)$, we can define the local utility index $U(x ; P)$ for any other outcome level $x$, by observing that

$$
P=\left(x_{1}, p_{1} ; \ldots ; x_{n}, p_{n}\right)=\left(x_{1}, p_{1} ; \ldots ; x_{n}, p_{n} ; x, 0\right)
$$

so that we can define 


$$
\left.U(x ; P) \equiv \frac{\partial \mho(P)}{\partial \operatorname{prob}(x)} \equiv \frac{\partial \nabla\left(x_{1}, p_{1} ; \ldots ; x_{n}, p_{n} ; x, p\right)}{\partial p}\right|_{p=0}
$$

Thus, $U(\because P)$ is really a local utility function over all outcome values $x$. In this more complete setting, the non-expected utility conditions for stochastic dominance preference, risk aversion, and comparative risk aversion are that at every $P$, the function $U(x ; P)$ must respectively be increasing in $x$, concave in $x$, and more concave in $x$-just like the conditions on $U(x)$ under expected utility theory. See Machina [1982, 1989], Chew, Epstein, and Zilcha [1988], and Karni [1987, 1989] for formal derivations and additional applications of this kind of analysis.

Although all of this says that the key to generalizing expected utility analysis is to think in terms of probability derivatives of the preference functional $\nabla(P)=\vartheta\left(x_{1}, p_{1} ; \ldots ; x_{n}, p_{n}\right)$, it is clear that the analysis of insurance and risk-sharing problems is going to have to involve its outcome derivatives as well. Fortunately, we can show that, as long as we continue to think of $U(x ; P)=(\partial \mathcal{P}(P) / \partial \operatorname{prob}(x))$ as the local utility function, the standard expected utility outcome derivative formula also generalizes ${ }^{11}$-that is,

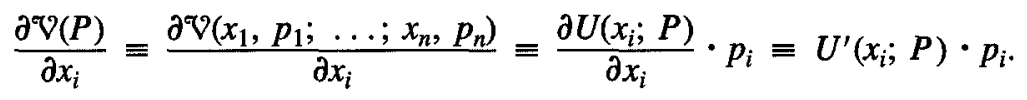

This gives us an immediate generalization of the expected utility $M R S$ formula for nonexpected utility indifference curves-namely,

$$
\operatorname{MRS}_{\vee}\left(x_{1}, x_{2}\right) \equiv-\frac{\partial \vee\left(x_{1}, \bar{p}_{1} ; x_{2}, \bar{p}_{2}\right) / \partial x_{1}}{\partial \vee\left(x_{1}, \bar{p}_{1} ; x_{2}, \bar{p}_{2}\right) / \partial x_{2}} \equiv-\frac{U^{\prime}\left(x_{1} ; P_{x_{1}, x_{2}}\right) \cdot \bar{p}_{1}}{U^{\prime}\left(x_{2} ; P_{x_{1}, x_{2}}\right) \cdot \bar{p}_{2}}
$$

where $P_{x_{1}, x_{2}}=\left(x_{1}, \bar{p}_{1} ; x_{2}, \bar{p}_{2}\right)$ is the probability distribution corresponding to the point $\left(x_{1}, x_{2}\right)$. It also gives us a generalization of the marginal expected utility formula-namely,

$$
\left.\frac{\partial \nabla\left(x_{1}+k, p_{1} ; \ldots ; x_{n}+k, p_{n}\right)}{\partial k}\right|_{k=0} \equiv \sum_{i=1}^{n} U^{\prime}\left(x_{i} ; P\right) \cdot p_{i}
$$

It should come as no surprise that formulas (15), (16), and (17) will come in very handy in checking the robustness of standard expected utility-based insurance theory.

$A$ settling of accounts. If a non-expected utility preference function $\nabla_{1}(\cdot)$ is at least as risk averse as another one $\nabla_{2}(\cdot)$, so that at each $P$ its local utility function $U_{1}(\bullet ; P)$ is at least as concave as $U_{2}(\because P)$, then the Arrow-Pratt theorem and the MRS formula (16) immediately imply the relative slope condition illustrated in Figure 5a. Similarly, the ArrowPratt theorem, the MRS formula (8), and a little calculus imply the relative slope condition illustrated in Figure 5b. Just as required! ${ }^{12}$

\section{Individual demand for insurance}

We now have a set of tools-graphical and algebraic-for representing and analyzing nonexpected utility risk preferences. I hope I've convinced you that the analysis of non-expected 
utility preferences is much closer to classical expected utility theory than you might have thought. Let's now turn toward applying these tools, in order to examine the robustness of standard insurance theory ${ }^{13}$ in the absence of the expected utility hypothesis.

Throughout the rest of this paper, we shall assume that risk preferences-expected utility or otherwise-are differentiable both in the outcomes and in the probabilities. In addition, since the results of insurance theory also almost all depend on the property of risk aversion, even under the expected utility hypothesis, there is no point in dropping that assumption when undertaking our non-expected utility examination. But as noted above, since risk aversion under expected utility also means outcome convexity, we could never be sure whether the result in question was really driven by risk aversion alone or by outcome convexity as well. ${ }^{14}$ Thus, when examining insurance theory in the absence of the expected utility hypothesis, our "robustness check" could reveal each expected utility-based insurance result to be in one of the following categories:

- The result only requires the assumption of risk aversion, without either outcome convexity or expected utility,

- The result requires outcome convexity (and hence also risk aversion) but not expected utility,

- The result simply doesn't hold at all without the expected utility hypothesis.

Naturally, when checking any given result, the higher up its category in this listing, the nicer it would be for non-expected utility theorists. And since robustness is a virtue, the nicer it would be for standard insurance theorists as well!

Throughout this section and its successors, we assume that the individual possesses an initial wealth level $w$ and faces the prospect of a random loss $\tilde{\ell}$, with probability distribution $\left(\ell_{1}, p_{1} ; \ldots ; \ell_{n}, p_{n}\right)$ (with each $\ell_{i} \geq 0$ ). An insurance policy consists of an indemnity function $I(\cdot)$ such that the individual receives payment $I(\ell)$ in the event of a loss of $\ell$, as well as a premium of $\pi$, which must be paid no matter what. Thus, the individual's random wealth on taking a policy (or contract) $(I(\cdot), \pi)$ becomes ${ }^{15}$

$$
w-\pi-\tilde{\ell}+I(\tilde{\ell})
$$

Of course, different forms of insurance involve different classes $\left\{\left(I_{\alpha}(\cdot), \pi_{\alpha}\right) \mid \alpha \in A\right\}$ of indemnity functions $I_{\alpha}(\cdot)$ and their corresponding premia $\pi_{\alpha}$, from which the individual may choose. In many cases, the premium for a given indemnity function $I(\cdot)$ takes the form $\pi=\lambda \cdot E[I(\tilde{\ell})]$, where $\lambda \geq 1$ is a loading factor. The results of standard insurance theory involve both characterization theorems and comparative statics theorems concerning individual maximization, bilateral efficiency, and group efficiency using the above framework.

For notational simplicity, we shall frequently work directly with random variables, such as $\tilde{\ell}$ or $w-\tilde{\ell}$, rather than with their probability distributions $\left(\ell_{1}, p_{1} ; \ldots ; \ell_{n}, p_{n}\right)$ or $\left(w-\ell_{1}, p_{1} ; \ldots ; w-\ell_{n}, p_{n}\right)$. In other words, given, say, a random variable $\tilde{x}$ with probability distribution $\left(x_{1}, p_{1} ; \ldots ; x_{n}, p_{n}\right)$, we shall use the term $\mathcal{V}(\tilde{x})$ as shorthand for $\nabla\left(x_{1}, p_{1} ; \ldots ; x_{n}, p_{n}\right)$. Thus, for example

$$
\nabla(w-\pi-\tilde{\ell}+I(\tilde{\ell})) \text { denotes } V\left(w-\pi-\ell_{1}+I\left(\ell_{1}\right), p_{1} ; \ldots ; w-\pi-\ell_{n}+I\left(\ell_{n}\right), p_{n}\right) \text {. }
$$




\subsection{Demand for coinsurance}

The very simplest results in insurance theory involve individual demand for a level $\alpha$ of coinsurance, given a fixed loading factor $\lambda \geq 1$. Formally, this setting consists of the set of policies $\left\{\left(I_{\alpha}(\cdot), \pi_{\alpha}\right) \mid \alpha \in[0,1]\right\}$, with

$$
\left.\begin{array}{rl}
\text { Indemnity function: } I_{\alpha}(\ell) \equiv \alpha \cdot \ell \\
\text { Premium: } \quad \pi_{\alpha}=\lambda \cdot \alpha \cdot E[\tilde{\ell}]
\end{array}\right\} \alpha \in[0,1] .
$$

In the expected utility framework, the individual's choice problem can therefore be written as

$$
\begin{aligned}
& \max _{\alpha \in[0,1]} E[U(w-\alpha \cdot \lambda \cdot E[\tilde{\ell}]-\tilde{\ell}+\alpha \cdot \tilde{\ell})] \text { or } \\
& \max _{\alpha \in[0,1]} E[U(w-\lambda \cdot E[\tilde{\ell}]-(1-\alpha) \cdot(\tilde{\ell}-\lambda \cdot E[\tilde{\ell}]))] .
\end{aligned}
$$

Denote the optimal choice in this problem by $\alpha^{*}$. This setting was studied early on, in classic papers by Borch [1961], Mossin [1968], and Smith [1968]. From the right side of (20), we see that a marginal change in insurance coverage $\alpha$ adds or subtracts the random variable $(\tilde{\ell}-\lambda \cdot E[\tilde{\ell}])$ to or from the individual's random wealth. Accordingly, we can term the variable $(\tilde{\ell}-\lambda \cdot E[\tilde{\ell}])$ the marginal insurable-risk variable.

The most basic analytical results for coinsurance are

CO.1. The first order condition for an interior optimum-a necessary condition for an interior global maximum-is that the expectation of the marginal-insurable-risk variable times the marginal utility of wealth is zero:

$$
E\left[(\tilde{\ell}-\lambda \cdot E[\tilde{\ell}]) \cdot U^{\prime}(w-\alpha \cdot \lambda \cdot E[\tilde{\ell}]-\tilde{\ell}+\alpha \cdot \tilde{\ell})\right]=0
$$

and under risk aversion, this is a sufficient condition for a global optimum.

CO.2. If the individual is risk averse, then full insurance will be demanded if and only if it is actuarially fair. In other words, $\alpha^{*}=1$ if and only if $\lambda=1$.

CO.3. If two risk averse individuals face the same choice problem except that the first is at least as risk averse as the second, then the first will demand at least as much insurance as the second. In other words, if $U_{1}\left({ }^{\circ}\right)$ is a concave transformation of $U_{2}(\cdot)$, then $\alpha_{1}^{*} \geq \alpha_{2}^{*} \cdot{ }^{16}$

Results CO.2 and CO.3 can both be illustrated in the Hirshleifer-Yaari diagram. ${ }^{17}$ Consider Figure $7 \mathrm{a}$, where the original uninsured position, point $A$, lies off the $45^{\circ}$ line, its corresponding full insurance point would lie exactly on the $45^{\circ}$ line, and the coinsurance "budget line" would connect the two points. The value $\alpha \in[0,1]$ corresponds to the position along the budget line from the uninsured point to the fully insured point. To see CO.2, note first that when insurance is actuarially fair, this budget line will correspond 


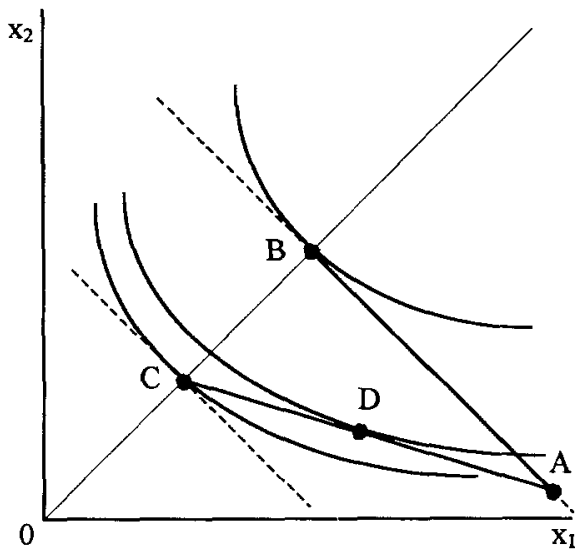

(a)

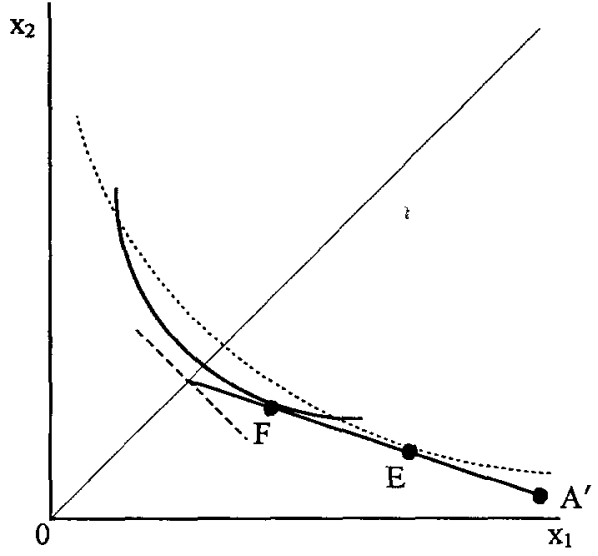

(b)

Figure 7. Optimal coinsurance (a) and effect of greater risk aversion on coinsurance (b) for risk averse expected utility preferences.

to the (dashed) iso-expected value line emanating from $A$, and risk aversion clearly implies that the optimal point on this line is its corresponding full-insurance point $B$. Next, note that when insurance is actuarially unfair, the budget line from $A$ is now flatter than the iso-expected value lines, so it is no longer tangent to the indifference curve through the (new) full-insurance point $C$. This implies that the new optimal point-namely, $D$-will involve less than full insurance. To see CO.3, consider Figure $7 \mathrm{~b}$ and recall from Figure 4a (or equations (2) and (12)) that for expected utility maximizers, the (solid) indifference curves of the more risk-averse person must be flatter than the (dotted) indifference curves of the less risk-averse one in the region below the $45^{\circ}$ line. This fact, coupled with the outcome-convexity property of risk-averse expected utility indifference curves, guarantees that, when both start from the same uninsured point $A^{\prime}$, the more risk-averse person will choose a greater level of coinsurance-point $F$ rather than point $E$.

How about the case of non-expected utility maximizers? In this case, the maximization problem for coinsurance (the indemnity/premium structure (19)) is

$$
\begin{aligned}
& \max _{\alpha \in[0,1]} \vartheta(w-\alpha \cdot \lambda \cdot E[\tilde{\ell}]-\tilde{\ell}+\alpha \cdot \tilde{\ell}) \text { or } \\
& \max _{\alpha \in[0,1]} \vartheta(w-\lambda \cdot E[\tilde{\ell}]-(1-\alpha) \cdot(\tilde{\ell}-\lambda \cdot E[\tilde{\ell}]))
\end{aligned}
$$

for some general non-expected utility preference function $\nabla(\cdot)$. Do any of the above expected utility-based results still hold? And if so, do they require just risk aversion, or do they also need outcome convexity?

To examine the robustness of CO.1, write (22) as $\max _{\alpha \in[0,1]} \nabla\left(w-\alpha \cdot \lambda \cdot E[\tilde{\ell}]-\ell_{1}+\alpha \cdot \ell_{1}, p_{1} ; \ldots ; w-\alpha \cdot \lambda \cdot E[\tilde{\ell}]-\ell_{n}+\alpha \cdot \ell_{n}, p_{n}\right)$. 
Formula (15) allows us to differentiate with respect to $\alpha$ to get the non-expected utility first-order condition

$$
\begin{gathered}
\frac{\partial \widetilde{V}\left(w-\alpha \cdot \lambda \cdot E[\tilde{\ell}]-\ell_{1}+\alpha \cdot \ell_{1}, p_{1} ; \ldots ; w-\alpha \cdot \lambda \cdot E[\tilde{\ell}]-\ell_{n}+\alpha \cdot \ell_{n}, p_{n}\right)}{\partial \alpha} \\
\quad=\sum_{i=1}^{n}\left(\ell_{i}-\lambda \cdot E[\tilde{\ell}]\right) \cdot U^{\prime}\left(w-\alpha \cdot \lambda \cdot E[\tilde{\ell}]-\ell_{i}+\alpha \cdot \ell_{i} ; P_{\alpha}\right) \cdot p_{i} \\
=E\left[(\tilde{\ell}-\lambda \cdot E[\tilde{\ell}]) \cdot U^{\prime}\left(w-\alpha \cdot \lambda \cdot E[\tilde{\ell}]-\tilde{\ell}+\alpha \cdot \tilde{\ell} ; P_{\alpha}\right)\right]=0
\end{gathered}
$$

where $P_{\alpha}$ denotes the wealth distribution $w-\alpha \cdot \lambda \cdot E[\tilde{\ell}]-\tilde{\ell}+\alpha \cdot \tilde{\ell}$ arising from the purchase of $\alpha$ coinsurance. This is precisely the analogue of the expected utility first-order condition (21) with the von Neumann-Morgenstern utility function $U(\cdot)$ replaced by the local utility function $U\left(\cdot ; P_{\alpha}\right)$ at the wealth distribution $P_{\alpha}{ }^{18}$ where

$$
P_{\alpha}=w-\alpha \cdot \lambda \cdot E[\tilde{\ell}]-\tilde{\ell}+\alpha \cdot \tilde{\ell} .
$$

Note that the necessity of condition (24) does not even require risk aversion, just differentiability. However, it should be clear from the Hirshleifer-Yaari diagram that it will only be sufficient under full outcome convexity. Otherwise, an indifference curve could be tangent to the budget line from below, and the point of tangency would be a (local or global) minimum.

Extending result CO.2 to the non-expected utility case is very straightforward and doesn't require outcome convexity at all. When insurance is actuarially fair $(\lambda=1)$, we have that for any $\alpha<1$, the random wealth

$$
w-\alpha \cdot E[\tilde{\ell}]-\tilde{\ell}+\alpha \cdot \tilde{\ell} \equiv w-E[\tilde{\ell}]-(1-\alpha) \cdot(\tilde{\ell}-E[\tilde{\ell}])
$$

differs from the full insurance $(\alpha=1)$ wealth of $w-E[\tilde{\ell}]$ by the addition of a zero-mean random variable. Accordingly, risk aversion alone implies that when coinsurance is actuarially fair, full coverage is optimal. Similarly, when insurance is unfair $(\lambda>1)$, we have that

$$
\begin{aligned}
\left.\frac{\partial V(w-\alpha \cdot \lambda \cdot E[\tilde{\ell}]-\tilde{\ell}+\alpha \cdot \tilde{\ell})}{\partial \alpha}\right|_{\alpha=1} & =E\left[(\tilde{\ell}-\lambda \cdot E[\tilde{\ell}]) \cdot U^{\prime}\left(w-\lambda \cdot E[\tilde{\ell}] ; P_{1}\right)\right] \\
& =(1-\lambda) \cdot E[\tilde{\ell}] \cdot U^{\prime}\left(w-\lambda \cdot E[\tilde{\ell}] ; P_{1}\right)<0,
\end{aligned}
$$

where $P_{1}$ is the degenerate distribution of the full-insurance wealth level $w-\lambda \cdot E[\tilde{\ell}]$. Thus, there will be values $\alpha<1$ that are strictly preferred to the full insurance position $\alpha=1$. This is all illustrated in Figure 8a, where indifference curves are risk averse but not outcome convex.

It would seem that if any of the coinsurance results depended crucially on the assumption of outcome convexity, it would be result CO.3, the result linking greater risk aversion to 


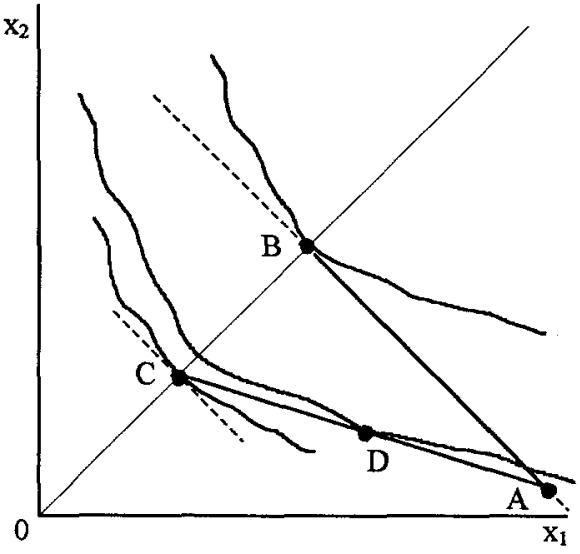

(a)

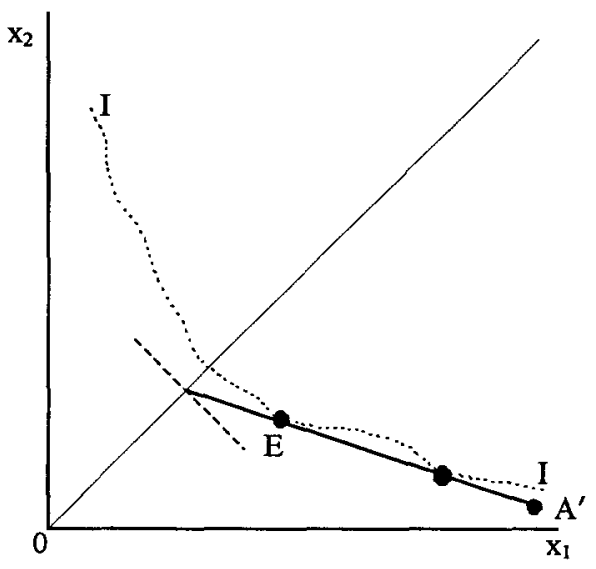

(b)

Figure 8. Optimal coinsurance (a) and effect of greater risk aversion on coinsurance (b) for non-expected utility preferences that are risk averse but not outcome convex.

greater coinsurance. This type of global comparative statics theorem is precisely the type of result we would expect to depend on the proper curvature of indifference curves, and a glance back at Figure $7 \mathrm{~b}$ would seem to reinforce this view. However, perhaps one of the most important points of this paper, which appears a few times, is that even for a result like this, outcome convexity is not needed.

The essence of this argument can be gleaned from Figure $8 \mathrm{~b}$. Recall that if preferences, though risk averse, are not outcome convex, then there is the possibility of multiple global optima, as with the dotted indifference curve in the figure. However, the essence of the comparative statics result $\mathrm{CO} .3$ is not that each individual must have a unique solution but that the less risk averse individual must always buy less insurance than the more risk averse individual.

To see that this still holds under non-expected utility, recall (from (12) and (16) or Figure 5a) that the non-expected utility condition for comparative risk aversion is that at each point below the $45^{\circ}$ line, the indifference curves of the more risk averse person are flatter than those of the less risk averse person. This means that any southeast movement along one of the less risk averse person's indifference curves must lower the preference function of the more risk averse person.

Now, to see that every optimum of the less risk averse person involves less insurance than every optimum of the more risk averse person, consider point $E$ in Figure $8 \mathrm{~b}$, which is that optimum for the less risk averse person that involves the most insurance for them, and consider their indifference curve through $E$ (call it $I-I$ ). Of course $I-I$ must lie everywhere on or above the insurance budget line. By the previous paragraph, the more risk averse person prefers $E$ to every point on $I-I$ that lies southeast of $E$ and hence (by the previous sentence) prefers $E$ to every point on the budget line that lies southeast of $E$. This then establishes that the very least amount of coinsurance that the more risk averse person would buy is at $E$. If the more risk averse person is in fact strictly more risk averse, the two persons' indifference curves cannot both be tangent to the budget line at $E$. Rather, 
the indifference curve of the more risk averse person will be flatter at that point, which implies that the least insurance they would ever buy is strictly more than the most insurance that the less risk averse person would ever buy (namely, $E$ ). Risk aversion (and comparative risk aversion) alone ensure this result, and outcome convexity is not needed at all. ${ }^{19}$

A formal algebraic statement of this result, which includes general probability distributions and allows for a corner solution (at zero insurance), is

Theorem 1. Let $w_{0}>0$ be base wealth, $\tilde{\ell} \geq 0$ be a random loss, and $\lambda>1$ a load factor, such that $w_{0}-\tilde{\ell}$ and $w_{0}-\lambda \cdot E[\tilde{\ell}]$ are both nonnegative. Assume that the nonexpected utility preference functions ${ }^{\circ} \mathcal{V}_{1}(\cdot)$ and $\boldsymbol{V}_{2}(\cdot)$ are twice continuously Frechet differentiable, strictly risk averse, and that ${ }^{\circ} \mathcal{1}_{1}(\cdot)$ is strictly more risk averse than $\nabla_{2}(\cdot)$ in the sense that $-U_{1}^{\prime \prime}(x ; F) / U_{1}^{\prime}(x ; F)>-U_{2}^{\prime \prime}(x ; F) / U_{2}^{\prime}(x ; F)$ for all $x$ and $F(\cdot)$. Consider the problem

$$
\max _{\alpha \in[0,1]} \vartheta_{i}\left(w_{0}-\alpha \cdot \lambda \cdot E[\tilde{\ell}]-\tilde{\ell}+\alpha \cdot \tilde{\ell}\right) \quad i=1,2 .
$$

If $\alpha_{1}^{*}$ is the smallest solution to this problem for $\nabla_{1}(\cdot)$, and $\alpha_{2}^{*}$ is the largest solution for $\nabla_{2}(\cdot)$, then $\alpha_{1}^{*} \geq \alpha_{2}^{*}$, with strict inequality unless $\alpha_{1}^{*}=0$.

Proof. In Appendix.

In other words, regardless of the possible multiplicity of optima due to non-outcome convexity, we will never observe the more risk averse first individual purchasing a smaller amount of insurance than the second individual, and the only time they would ever purchase the same amount is if the terms are so unattractive that zero insurance is an optimum even for the first individual, in which case it is the only optimum for the second individual.

To sum up our robustness check on the basic theory of coinsurance: except for the additional status of the necessary condition (21) as a sufficient condition as well (which also requires outcome convexity), all three of the coinsurance results CO.1, CO.2, and CO.3 generalize to the case of non-expected utility preferences under the assumption of simple risk aversion alone. In other words, at least at this most basic level, the standard theory of demand for coinsurance is, happily, extremely robust.

\subsection{Demand for deductible insurance}

A second type of insurance contract, distinct from the coinsurance contract considered above, is deductible insurance. Given a fixed actuarial loading factor $\lambda \geq 1$, this setting consists of the set of contracts $\left\{\left(I_{\alpha}(\cdot), \pi_{\alpha}\right) \mid \alpha \in[0, M]\right\}$, where $M$ is the largest possible value of the loss $\ell$, and

$$
\left.\begin{array}{rl}
\text { Indemnity function: } I_{\alpha}(\ell) \equiv \max \{\ell-\alpha, 0\} \\
\text { Premium: } \quad \pi_{\alpha}=\lambda \cdot E\left[I_{\alpha}(\tilde{\ell})\right]
\end{array}\right\} \alpha \in[0, M] .
$$


In the expected utility framework, the individual's choice problem can therefore be written as

$$
\begin{gathered}
\max _{\alpha \in[0, M]} E\left[U\left(w-\lambda \cdot E\left[I_{\alpha}(\tilde{\ell})\right]-\tilde{\ell}+\max \{\tilde{\ell}-\alpha, 0\}\right)\right] \text { or } \\
\max _{\alpha \in[0, M]} E[U(w-\lambda \cdot E[\max \{\tilde{\ell}-\alpha, 0\}]-\min \{\tilde{\ell}, \alpha\})] .
\end{gathered}
$$

Once again, denote the optimal choice by $\alpha^{*}$. This problem has been studied by, among others, Mossin [1968], Gould [1969], Pashigian, Schkade, and Menefee [1966], Moffet [1977], Schlesinger [1981], Drèze [1981], Karni [1983, 1985], and Eeckhoudt, Gollier, and Schlesinger [1991].

The insurance budget line for this problem in the case of two states is illustrated in the Hirshleifer-Yaari diagram of Figure 9. Given an initial (preloss) wealth point $W=(w, w)$, the uninsured point $A$ reflects a small loss $\ell_{1}$ in state 1 and a larger loss $\ell_{2}$ in state 2 . The thick line in the figure represents the kinked insurance budget line when insurance is actuarially unfair (otherwise, is it simply the dashed iso-expected value line through $A$ ). Starting at the deductible level $\alpha=\ell_{2}$ (no insurance) each unit drop in $\alpha$ lowers wealth in state 1 by the premium $\lambda \cdot \bar{p}_{2}$ and raises wealth in state 2 by $1-\lambda \cdot \bar{p}_{2}$, while lowering the overall expected value of wealth. This generates a linear budget line from point $A$ to the certainty line at point $B$, where $\alpha$ has dropped by $\left(\ell_{2}-\ell_{1}\right)$ (so now $\alpha=\ell_{1}$ ), and the individual's wealth is equal to $w-\ell_{1}-\lambda \cdot \bar{p}_{2} \cdot\left(\ell_{2}-\ell_{1}\right)$ in each state. Note that while a still smaller deductible $\alpha<\ell_{1}$ is possible, this is basically further insuring what is now a sure prospect, and doing so at actuarially unfair rates, so it moves the individual down the $45^{\circ}$ line. In the limit, when $\alpha=0$, wealth in each state would be $w-\lambda \cdot\left(\bar{p}_{1} \cdot \ell_{1}+\bar{p}_{2} \cdot \ell_{2}\right)$ (point $C$ ).

The point of presenting Figure 9 is to show that, for the two-state case, the budget line for deductible insurance (at least the relevant part $A-B$ ) is so similar to the budget line for

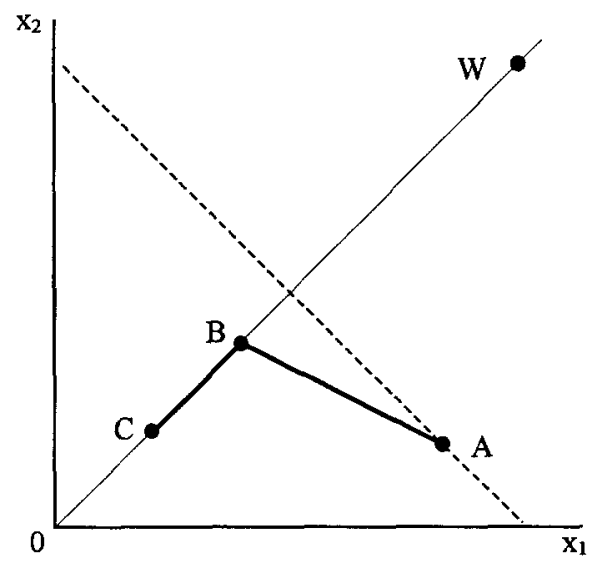

Figure 9. Insurance budget line for deductible insurance. 
coinsurance that all of the graphical intuition obtained from Figures 7 and 8 concerning coinsurance will carry over to Figure 9 and to deductible insurance. But given the fact that most of the "action" of the deductible problem (30) occurs in the case of a multitude (or continuum) of states, we do not repeat the graphical analyses of Figures 7 and 8 here.

Rather, we proceed directly to our algebraic robustness check. To avoid the types of kinks that occur as $\alpha$ crosses the value of some discrete loss value $\ell_{i}$, we assume that the random variable $\tilde{\ell}$ has a continuous cumulative distribution function $F(\cdot)$ with support $[0, M]$. We consider the corresponding basic results for deductible insurance:

$D E .1$. The first-order condition for an interior optimum (that is, the necessary condition for an interior global maximum) is

$$
\begin{aligned}
E[[\lambda \cdot(1-F(\alpha))-\operatorname{sgn}(\max \{\tilde{\ell}-\alpha, 0\})] \\
\left.\cdot U^{\prime}(w-\lambda \cdot E[\max \{\tilde{\ell}-\alpha, 0\}]-\min \{\tilde{\ell}, \alpha\})\right]=0
\end{aligned}
$$

where $\operatorname{sgn}(z)=+1 / 0 /-1$ as $z>/=/<0.20$

$D E$.2. If the individual is risk averse, then full insurance will be demanded if and only if it is actuarially fair. In other words, $\alpha^{*}=0$ if and only if $\lambda=1$.

$D E .3$. If two risk-averse individuals face the same choice problem except that the first is at least as risk averse as the second, then the first will demand at least as much insurance as (i.e., have a lower deductible than) the second. In other words, if $U_{1}(\cdot)$ is a concave transformation of $U_{2}(\cdot)$, then $\alpha_{1}^{*} \leq \alpha_{2}^{*}{ }^{21}$

The non-expected utility version of the deductible problem (30) is

$$
\begin{gathered}
\max _{\alpha \in[0, M]} \nabla\left(w-\lambda \cdot E\left[I_{\alpha}(\tilde{\ell})\right]-\tilde{\ell}+\max \{\tilde{\ell}-\alpha, 0\}\right) \text { or } \\
\max _{\alpha \in[0, M]} \nabla(w-\lambda \cdot E[\max \{\tilde{\ell}-\alpha, 0\}]-\min \{\tilde{\ell}, \alpha\}) .
\end{gathered}
$$

Formula (15) allows us to differentiate these objective functions with respect to $\alpha$, to get the non-expected utility first order condition

$$
\begin{aligned}
\int_{0}^{M}[\lambda \cdot(1-F(\alpha))-\operatorname{sgn}(\max \{\tilde{\ell}-\alpha, 0\})] \\
\quad \cdot U^{\prime}\left(w-\lambda \cdot E[\max \{\tilde{\ell}-\alpha, 0\}]-\min \{\ell, \alpha\} ; F_{\alpha}\right) \cdot d F(\ell)=0,
\end{aligned}
$$

where $F_{\alpha}(\cdot)$ is the distribution of the random variable $w-\lambda \cdot E[\max \{\tilde{\ell}-\alpha, 0\}]-$ $\min \{\tilde{\ell}, \alpha\}$. This is once again seen to be equivalent to the expected utility first order condition (31), with the von Neumann-Morgenstern utility function $U(\cdot)$ replaced by the local utility function $U\left(\bullet ; F_{\alpha}\right)$ at the distribution $F_{\alpha}\left({ }^{\circ}\right)$ implied by the optimal choice. Thus, DE.1 generalizes to non-expected utility.

The "if" part of result DE.2-namely, full insurance under actuarial fairness-follows immediately from risk aversion without outcome convexity, just as it did in the case of 
coinsurance. To see that the "only if" part does not require outcome convexity either, consider the case $\lambda>1$ and evaluate the left side of (33) at the full-insurance point $\alpha=0$, to obtain

$\left.\frac{\partial \nabla(w-\lambda \cdot E[\max \{\tilde{\ell}-\alpha, 0\}]-\min \{\tilde{\ell}, \alpha\})}{\partial \alpha}\right|_{\alpha=0}=[\lambda-1] \cdot U^{\prime}\left(w-\lambda \cdot E[\tilde{\ell}] ; F_{0}\right)>0$,

where $F_{0}(\cdot)$ is the degenerate distribution of the full-insurance wealth level $w-\lambda \cdot E[\tilde{\ell}]$. Thus, in this case there will be values $\alpha>1$ that are strictly preferred to the full-insurance level $\alpha=0$.

Finally, the comparative static result DE.3: as it turns out, the argument behind Figure $8 \mathrm{~b}$ (more correctly, the argument behind Theorem 1) applies to the case of deductible insurance as well. We have

Theorem 2. Let $w_{0}>0$ be base wealth, let $\tilde{\ell}$ be a random loss with support $[0, M]$ $\left(M<w_{0}\right)$ and continuous cumulative distribution function $F_{\tilde{\ell}}(\cdot)$, and let $\lambda>1$ be a load factor. Assume that the non-expected utility preference functions $\nabla_{1}(\cdot)$ and $\vartheta_{2}(\cdot)$ are twice continuously Fréchet differentiable, strictly risk averse, and that $\nabla_{1}\left({ }^{\circ}\right)$ is strictly more risk averse than $\nabla_{2}\left({ }^{\circ}\right)$ in the sense that $-U_{1}^{\prime \prime}(x ; F) / U_{1}^{\prime}(x ; F)>-U_{2}^{\prime \prime}(x ; F) / U_{2}^{\prime}(x ; F)$ for all $x$ and $F(\cdot)$. Consider the problem

$$
\max _{\alpha \in[0, M]} \mathscr{V}_{i}\left(w_{0}-\lambda \cdot E[\max \{\tilde{\ell}-\alpha, 0\}]-\tilde{\ell}+\max \{\tilde{\ell}-\alpha, 0\}\right) \quad i=1,2 .
$$

If $\alpha_{1}^{*}$ is the largest solution to this problem for $\nabla_{1}(\cdot)$, and $\alpha_{2}^{*}$ is the smallest solution for $\nabla_{2}(\cdot)$, then $\alpha_{1}^{*} \leq \alpha_{2}^{*}$, with strict inequality unless $\alpha_{1}^{*}=M$.

Proof. In Appendix.

In other words, regardless of the possible multiplicity of optima due to non-outcome convexity, we will never observe the more risk averse first individual choosing a higher level of deductible (less insurance) than the second individual, and the only time they would ever choose the same level is if the terms are so unattractive that no insurance $(\alpha=M)$ is an optimum even for the first individual, in which case it is the only optimum for the second individual.

Perhaps surprisingly, or perhaps not, our robustness findings for at least the most basic aspects of deductible insurance parallel those of coinsurance: except for the additional status of condition (31) as a sufficient condition (which requires outcome convexity), all of the deductible results DE.1, DE.2, and DE.3 generalize to the case of non-expected utility preferences under the assumption of risk aversion alone.

\section{Pareto-efficient bilateral insurance contracts}

The results of the previous section have examined the customer's optimal amount of insurance, taking the form of the insurance contract (either coinsurance or deductible) as given. 
However, an important set of results in insurance theory attempts to determine the optimal (Pareto-efficient) form of insurance contract, given the nature of the insurer's costs and risk preferences. Will these results be robust to dropping the expected utility hypothesis?

The basic theorems on Pareto-efficient bilateral insurance contracts concern the case where the insurer possesses an increasing cost function $C(I)$ for indemnity payments $I \geq 0$. These costs include the indemnity payment itself plus any additional processing or transactions costs. In the expected utility case, a Pareto-efficient contract $(I(\cdot), \pi)$ can be represented as the solution to

$$
\begin{aligned}
& \max _{I(\cdot), \pi} E\left[U_{1}\left(w_{1}-\pi-\tilde{\ell}+I(\tilde{\ell})\right)\right] \\
& \text { s.t.: }\left\{\begin{array}{c}
E\left[U_{2}\left(w_{2}+\pi-C(I(\tilde{\ell}))\right)\right]=U_{2}\left(w_{2}\right) \\
0 \leq I(\ell) \leq \ell,
\end{array}\right.
\end{aligned}
$$

where $U_{1}(\cdot)$ is the concave utility function of the insured, $U_{2}(\cdot)$ is the utility function of the insurer, and $w_{1}$ and $w_{2}$ are their respective initial wealth levels. The loss variable $\tilde{\ell}$ is assumed to have a continuous cumulative distribution function $F_{\tilde{\ell}}(\cdot)$ over some interval $[0, M]$.

Arrow [1963, app. $]^{22}$ considered the simplest case where the cost function takes the linear form $C(I) \equiv \lambda \cdot I$ (for $\lambda>1$ ), and the insurer is risk neutral. Under these assumptions, the upper constraint in (36) immediately implies the standard loading formula

$$
\pi=\lambda \cdot E[I(\tilde{\ell})],
$$

and Arrow showed that the Pareto-efficient indemnity function $I(\cdot)$ must take the deductible form

$$
I(\ell) \equiv \min \{\ell-\alpha, 0\} .
$$

Needless to say, this forms an important justification for studying the individual's demand for insurance under the deductible structure, as we did in Section 3.2.

This result has been extended in a few directions by Raviv [1979], so that we can now consider the set of expected utility-based results:

$P E .1$. Given risk neutrality of the insurer and a linear cost function (with $\lambda>1$ ), the Pareto-efficient bilateral insurance contract must take the deductible form (38), for a positive deductible $\alpha$.

$P E$.2. Given strict risk aversion of the insurer and a linear cost function (with $\lambda>1$ ), the Pareto-efficient bilateral insurance contract must take the form of coinsurance above a nonnegative deductible $\alpha$-that is,

$$
\begin{aligned}
I(\ell)=0 & \text { for } \ell \leq \alpha \\
0<I(\ell)<\ell & \text { for } \ell>\alpha \\
0<I^{\prime}(\ell)<1 & \text { for } \ell>\alpha .
\end{aligned}
$$


$P E$.3. Given risk neutrality of the insurer and a strictly convex cost function $C(\cdot)\left(C^{\prime \prime}(\cdot)\right.$ $>0$ ), the Pareto-efficient bilateral insurance contract must again take the form of coinsurance above a deductible, as in (39), where the deductible $\alpha$ is strictly positive.

Just as Arrow's original result (PE.1) gave a justification for the study of deductibles, the results PE. 2 and PE. 3 provide a justification for the study of the demand for coinsurance as we undertook in Section 3.1.23

Do these results extend to non-expected utility maximizers, and if so, is risk aversion sufficient to obtain them, or do we also need to assume outcome convexity? Under nonexpected utility, the Pareto-efficient contracts are characterized by the solutions to

$$
\begin{aligned}
& \max _{I(\cdot), \pi} \nabla_{1}\left(w_{1}-\pi-\tilde{\ell}+I(\tilde{\ell})\right) \\
& \text { s.t.: }\left\{\begin{array}{c}
\nabla_{2}\left(w_{2}+\pi-C(I(\tilde{\ell}))\right)=\nabla_{2}\left(w_{2}\right) \\
0 \leq I(\ell) \leq \ell .
\end{array}\right.
\end{aligned}
$$

Concerning PE.1, note that under its assumptions, the standard loading formula (37) continues to follow from the constraint in (40). In such a case, Karni [1992] has proven that, given differentiability of $\mathcal{V}_{1}(\cdot)$, risk aversion alone ensures that any Pareto-efficient insurance contract must continue to take the pure deductible form (38). More recently, Gollier and Schlesinger [1995] have provided an ingenious proof of PE.1 based solely on risk aversion considerations and hence similarly independent of the expected utility hypothesis.

The robustness of PE. 2 and PE. 3 to non-expected utility can be demonstrated by using the same type of proof that Karni used to generalize PE.1. We present an informal sketch here. Let $\left(I^{*}(\cdot), \pi^{*}\right)$ be a Pareto-efficient insurance contract between $\nabla_{1}(\cdot)$ (which is risk averse) and $\nabla_{2}(\cdot)$, under the assumptions of either PE.2 or PE. $3 .^{24}$ In such a case, no joint differential change ${ }^{25}(\Delta I(\cdot), \Delta \pi)$ from $\left(I^{*}(\cdot), \pi^{*}\right)$ that continues to satisfy the conditions $\nabla_{2}\left(w_{2}+\pi-C(I(\tilde{\ell}))\right)=\nabla_{2}\left(w_{2}\right)$ and $0 \leq I(\ell) \leq \ell$ should be able to raise the value of $\nabla_{1}\left(w_{1}-\pi-\tilde{\ell}+I(\tilde{\ell})\right)$. However, from the cumulative distribution function version of (15), the effect of any such differential change $(\Delta I(\cdot), \Delta \pi)$ from $\left(I^{*}(\cdot), \pi^{*}\right)$ on the value of $\vartheta_{1}\left(w_{1}-\pi-\tilde{\ell}+I(\tilde{\ell})\right)$ is given by the expression

$$
\int_{0}^{M} U_{1}^{\prime}\left(w_{1}-\pi^{*}-\ell+I^{*}(\ell) ; F_{w_{1}-\pi^{*}-\tilde{\ell}+\tilde{I}^{*}(\tilde{\ell})}\right) \cdot[\Delta I(\ell)-\Delta \pi] \cdot d F_{\tilde{\ell}}(\ell),
$$

and similarly, the effect of any differential change $(\Delta I(\cdot), \Delta \pi)$ from $\left(I^{*}(\cdot), \pi^{*}\right)$ on the value of $\nabla_{2}\left(w_{2}+\pi-C(I(\tilde{\ell}))\right)$ is given by

$\int_{0}^{M} U_{2}^{\prime}\left(w_{2}+\pi^{*}-C\left(I^{*}(\ell)\right) ; F_{w_{2}+\pi^{*}-C\left(I^{*}(\tilde{\ell})\right)}\right) \cdot\left[\Delta \pi-C^{\prime}\left(I^{*}(\ell)\right) \cdot \Delta I(\ell)\right] \cdot d F_{\tilde{\ell}}(\ell)$.

Thus, any solution $\left(I^{*}(\cdot), \pi^{*}\right)$ to (40) must satisfy the property:

"No differential change $(\Delta I(\cdot), \Delta \pi)$ that makes (42) equal to zero can make (41) positive." 
However, this is precisely the statement that the contract $\left(I^{*}(\cdot), \pi^{*}\right)$ satisfies the first-order conditions for the expected utility problem (36), for the fixed von Neumann-Morgenstern utility functions $U_{1}\left({ }^{*}\right)=U_{1}\left(\cdot ; F_{w_{1}-\pi^{*}-\tilde{\ell}+I^{*}(\tilde{\ell})}\right.$ (which is concave) and $U_{2}\left({ }^{*}\right)=U_{2}(* ;$ $F_{w_{2}+\pi^{*}-C\left(I^{*}(\tilde{\ell})\right)}$ ) (which under PE.2 is also concave), and we know from the expected utility versions of PE. 2 and PE. 3 that any pair $(I(\cdot), \pi)$ that satisfies these first-order conditions, including therefore the pair $\left(I^{*}(\cdot), \pi^{*}\right)$, must satisfy the "coinsurance above a deductible" condition (39). Furthermore, under the assumptions of PE.3, they must satisfy the additional property that the deductible is positive. Note that, like Karni, we needed to assume risk aversion of $\nabla_{1}\left({ }^{\circ}\right)$ (and also $\nabla_{2}\left({ }^{\circ}\right)$ for PE.2) but not outcome convexity. ${ }^{26}$

Thus another set of basic results in insurance theory seem to be quite robust to dropping the expected utility hypothesis.

\section{Pareto-efficient multilateral risk sharing}

An important part of the theory of insurance is the joint risk sharing behavior of a group of individuals. Research in this area was first initiated by Borch [1960, 1961, 1962] and Wilson [1968], and the modern theory of insurance markets can truly be said to stem from these papers. ${ }^{27}$

Under expected utility, this framework consists of a set $\{\theta\}$ of states of nature, and $n$ individuals, each with von Neumann-Morgenstern utility function $U_{i}\left(\bullet^{\circ}\right)$ and random endowment $w_{i}(\theta)$. In this paper, we consider the special case where there are a finite number of states $\left\{\theta_{1}, \ldots, \theta_{T}\right\}$, and where agents agree ${ }^{28}$ on their probabilities $\left\{\operatorname{prob}\left(\theta_{1}\right), \ldots, \operatorname{prob}\left(\theta_{T}\right)\right\}$ (all positive). A risk sharing rule is a set of functions $\left\{s_{i}(\cdot) \mid i=1, \ldots, n\right\}$ that determines person $i$ 's allocation as a function of the state of nature. Under such a rule, person $i$ 's expected utility is accordingly given by

$$
\sum_{t=1}^{T} U_{i}\left(s_{i}\left(\theta_{t}\right)\right) \cdot \operatorname{prob}\left(\theta_{t}\right)
$$

A risk sharing rule $\left\{s_{i}(\cdot) \mid i=1, \ldots, n\right\}$ is feasible if it satisfies the constraint

$$
\sum_{i=1}^{n} s_{i}\left(\theta_{t}\right) \underset{\theta_{t}}{\equiv} \sum_{i=1}^{n} w_{i}\left(\theta_{t}\right)
$$

and it is Pareto-efficient if there exists no other feasible rule that preserves or increases the expected utility of each member, with a strict increase for at least one member. Finally, define the risk tolerance measure ${ }^{29}$ of a utility function $U_{i}\left({ }^{\circ}\right)$ by

$$
\rho_{i}(x) \underset{x}{\equiv}-U_{i}^{\prime}(x) / U_{i}^{\prime \prime}(x)
$$

Given this framework, three of the most basic analytical results for Pareto-efficient risk sharing are 
RS.1. A necessary condition for a risk sharing rule $\left\{s_{i}(\cdot) \mid i=1, \ldots, n\right\}$ to be Paretoefficient is that there exists a set of nonnegative weights $\left\{\lambda_{1}, \ldots, \lambda_{n}\right\}$ such that

$$
\lambda_{i} \cdot U_{i}^{\prime}\left(s_{i}\left(\theta_{t}\right)\right) \underset{\theta_{t}}{\equiv} \lambda_{j} \cdot U_{j}^{\prime}\left(s_{j}\left(\theta_{t}\right)\right) \quad i, j=1, \ldots, n
$$

and under risk aversion, this is a sufficient condition.

RS.2. Any Pareto-efficient risk-sharing rule will satisfy the mutuality principle (e.g., Gollier [1992, p. 7])-namely, that the share $s_{i}\left(\theta_{t}\right)$ depends upon the state of nature $\theta_{t}$ only through the total group endowment $w\left(\theta_{t}\right) \equiv \Sigma_{k=1}^{n} w_{k}\left(\theta_{t}\right)$ in state $\theta_{t}$. In other words, there exists functions $\left\{x_{i}(\cdot) \mid i=1, \ldots, n\right\}$ such that

$$
s_{i}\left(\theta_{t}\right) \underset{\theta_{t}}{\stackrel{\equiv}{\theta_{i}}} x_{i}\left(w\left(\theta_{t}\right)\right) \quad i=1, \ldots, n .
$$

$R S .3$. In the case of a continuum of states, the members' incremental shares $x_{i}^{\prime}(w)$ are proportional to their respective risk tolerances, evaluated along the optimal sharing rule:

$$
x_{i}^{\prime}(w) \underset{w}{\equiv} \frac{\rho_{i}\left(x_{i}(w)\right)}{\Sigma_{k=1}^{n} \rho_{k}\left(x_{k}(w)\right)} \quad i=1, \ldots, n
$$

Do these results extend to non-expected utility? To check, take a set of $\boldsymbol{n}$ non-expected utility maximizers with preference functions $\left\{\nabla_{1}\left({ }^{\circ}\right), \ldots, \nabla_{n}(\cdot)\right\}$. The natural generalization of condition (46) would be that there exists a set of nonnegative weights $\left\{\lambda_{1}, \ldots, \lambda_{n}\right\}$ such that

$$
\lambda_{i} \cdot U_{i}^{\prime}\left(s_{i}(\theta) ; P_{i}^{*}\right) \underset{\theta}{\equiv} \lambda_{j} \cdot U_{j}^{\prime}\left(s_{j}(\theta) ; P_{j}^{*}\right) \quad i, j=1, \ldots, n,
$$

where $U_{i}(\cdot ; P)$ and $U_{j}(\because P)$ are the local utility functions of $\vartheta_{i}(\cdot)$ and $\vartheta_{j}(\cdot)$, and $P_{i}^{*}$ and $P_{j}^{*}$ are the probability distributions of the random variables $s_{i}\left(\tilde{\theta}_{t}\right)$ and $s_{j}\left(\tilde{\theta}_{t}\right)$, respectively. To check the robustness of RS.1, assume (49) did not hold, in which case there would be some states $\theta_{a}, \theta_{b}$, and individuals $i, j$ such that

$$
\frac{U_{i}^{\prime}\left(s_{i}\left(\theta_{a}\right) ; P_{i}^{*}\right)}{U_{i}^{\prime}\left(s_{i}\left(\theta_{b}\right) ; P_{i}^{*}\right)} \neq \frac{U_{j}^{\prime}\left(s_{j}\left(\theta_{a}\right) ; P_{j}^{*}\right)}{U_{j}^{\prime}\left(s_{j}\left(\theta_{b}\right) ; P_{j}^{*}\right)}
$$

and hence

$$
\frac{U_{i}^{\prime}\left(s_{i}\left(\theta_{a}\right) ; P_{i}^{*}\right)}{U_{i}^{\prime}\left(s_{i}\left(\theta_{b}\right) ; P_{i}^{*}\right)} \cdot \frac{\operatorname{prob}\left(\theta_{a}\right)}{\operatorname{prob}\left(\theta_{b}\right)} \neq \frac{U_{j}^{\prime}\left(s_{j}\left(\theta_{a}\right) ; P_{j}^{*}\right)}{U_{j}^{\prime}\left(s_{j}\left(\theta_{b}\right) ; P_{j}^{*}\right)} \cdot \frac{\operatorname{prob}\left(\theta_{a}\right)}{\operatorname{prob}\left(\theta_{b}\right)}
$$

But from the $n$-state version of the $M R S$ formula (16), ${ }^{30}$ this means that the two individuals' marginal rates of substitution between consumption in states $\theta_{a}$ and $\theta_{b}$ are strictly unequal, which gives them an opportunity for mutually beneficial trade. Thus, the original sharing rule was not Pareto-efficient. This establishes that (49) is indeed a necessary condition for Pareto-efficiency. A standard Edgeworth box argument will establish that it will also be a sufficient condition provided outcome convexity holds, though not otherwise. 
To check result RS.2, observe that if it did not hold, there would be two states $\theta_{a}, \theta_{b}$ and an individual $i$ such that $\Sigma_{k=1}^{n} w_{k}\left(\theta_{a}\right)=\Sigma_{k=1}^{n} w_{k}\left(\theta_{b}\right)$, but $s_{i}\left(\theta_{a}\right)>s_{i}\left(\theta_{b}\right)$. But by the feasibility condition (44), this means that there must exist some other individual $j$ such that $s_{j}\left(\theta_{a}\right)<s_{j}\left(\theta_{b}\right)$. By risk aversion (and hence concavity of each individual's local utility function), this would then imply

$$
\frac{U_{i}^{\prime}\left(s_{i}\left(\theta_{a}\right) ; P_{i}^{*}\right)}{U_{i}^{\prime}\left(s_{i}\left(\theta_{b}\right) ; P_{i}^{*}\right)} \cdot \frac{\operatorname{prob}\left(\theta_{a}\right)}{\operatorname{prob}\left(\theta_{b}\right)}<\frac{\operatorname{prob}\left(\theta_{a}\right)}{\operatorname{prob}\left(\theta_{b}\right)}<\frac{U_{j}^{\prime}\left(s_{j}\left(\theta_{a}\right) ; P_{j}^{*}\right)}{U_{j}^{\prime}\left(s_{j}\left(\theta_{b}\right) ; P_{j}^{*}\right)} \cdot \frac{\operatorname{prob}\left(\theta_{a}\right)}{\operatorname{prob}\left(\theta_{b}\right)},
$$

so that, as before, the two individuals have different marginal rates of substitution between consumption in states $\theta_{a}$ and $\theta_{b}$, so the original sharing rule could not have been Paretoefficient. Thus, the mutuality principle (result RS.2) and the formula (47) hold for nonexpected utility risk sharers. Note: Only risk aversion, and not outcome convexity, is needed for this result.

Finally, to show that the continuum-state-space result RS.3 also generalizes, combine (47) and (49) (which both continue to hold with a continuum of states) to write

$$
\lambda_{i} \cdot U_{i}^{\prime}\left(x_{i}(w) ; F_{i}^{*}\right) \underset{w}{\equiv} \lambda_{j} \cdot U_{j}^{\prime}\left(x_{j}(w) ; F_{j}^{*}\right) \quad i, j=1, \ldots, n
$$

where $F_{i}^{*}\left({ }^{\circ}\right)$ and $F_{j}^{*}(\cdot)$ are the cumulative distribution functions of the (continuous) random variables $s_{i}(\theta)$ and $s_{j}(\theta)$ (see note 12 ). Differentiating (53) with respect to $w$ and then dividing by (53) yields

$$
\frac{U_{i}^{\prime \prime}\left(x_{i}(w) ; F_{i}^{*}\right)}{U_{i}^{\prime}\left(x_{i}(w) ; F_{i}^{*}\right)} \cdot x_{i}^{\prime}(w) \equiv \frac{U_{j}^{\prime \prime}\left(x_{j}(w) ; F_{j}^{*}\right)}{U_{j}^{\prime}\left(x_{j}(w) ; F_{j}^{*}\right)} \cdot x_{j}^{\prime}(w) \quad i, j=1, \ldots, n
$$

and hence

$$
\frac{\rho_{j}\left(x_{j}(w) ; F_{j}^{*}\right)}{\rho_{i}\left(x_{i}(w) ; F_{i}^{*}\right)} \cdot x_{i}^{\prime}(w) \equiv \underset{w}{\equiv} x_{j}^{\prime}(w) \quad i, j=1, \ldots, n,
$$

where $\rho_{i}(x ; F) \equiv-U_{i}^{\prime}(x ; F) / U_{i}^{\prime \prime}(x ; F)$ is the risk-tolerance measure of the local utility function $U_{i}(\because ; F)$. Summing over $j=1, \ldots, n$, noting that the feasibility constraint implies $\Sigma_{j=1}^{n} x_{j}^{\prime}(w) \underset{w}{\equiv} 1$, and solving finally yields

$$
x_{i}^{\prime}(w) \equiv \frac{\rho_{i}\left(x_{i}(w) ; F_{i}^{*}\right)}{\sum_{k=1}^{n} \rho_{k}\left(x_{k}(w) ; F_{k}^{*}\right)} \quad i=1, \ldots, n \text {. }
$$

In other words, each member's incremental share is proportional to their local risk tolerance, evaluated along the optimal sharing rule. (Recall that since $F_{1}^{*}, \ldots, F_{n}^{*}$ are the probability distributions of $s_{1}(\theta), \ldots, s_{n}(\theta)$, they are determined directly by the optimal sharing rule.)

What does this all imply? It is true that we need outcome convexity to guarantee the sufficiency of the Pareto-efficiency condition (49). However, it remains a necessary property of any Pareto-efficient allocation even without outcome convexity. Otherwise, risk aversion alone (and sometimes not even that) suffices to generalize the basic risk sharing results RS.1, RS.2, and RS.3 to the case of non-expected utility maximizers. 


\section{Self-insurance versus self-protection}

Our final topic stems from the seminal article of Ehrlich and Becker [1972], who examined two important nonmarket risk-reduction activities-namely, self-insurance, where resources are expended to reduce the magnitude of a possible loss, and self-protection where resources are expended to reduce the probability of that loss. In a two-state framework (the one they considered), the individual's initial position can be represented as the probability distribution $(w-\ell, p ; w, 1-p)$-that is to say, base wealth $w$ with a $p$ chance of a loss of $\ell$.

The possibility of self-insurance can be represented by an expenditure variable $\alpha \in[0, M]$, such that the first-state loss becomes $\ell(\alpha)$, where $\ell^{\prime}(\alpha)<0$. In that case, an expected utility maximizer's decision problem is

$$
\max _{\alpha \in[0, M]}[p \cdot U(w-\ell(\alpha)-\alpha)+(1-p) \cdot U(w-\alpha)] .
$$

On the other hand, self-protection can be represented by an expenditure variable $\beta \in[0, M]$, such that the probability of a loss becomes $p(\beta)$, where $p^{\prime}(\beta)<0$. In that case, an expected utility maximizer's decision problem is

$$
\max _{\beta \in[0, M]}[p(\beta) \cdot U(w-\ell-\beta)+(1-p(\beta)) \cdot U(w-\beta)] .
$$

Needless to say, these activities could be studies in conjunction with each other, as well as in conjunction with market insurance, and Ehrlich and Becker do precisely that. Since then, the self-insurance and self-protection framework (with or without market insurance) has been extensively studied (see, for example, Boyer and Dionne [1983, 1989], Dionne and Eeckhoudt [1985], Chang and Ehrlich [1985], Hibert [1989], Briys and Schlesinger [1990], Briys, Schlesinger, and Schulenburg [1991], and Sweeney and Beard [1992]).

Konrad and Skaperdas [1993] examine self-insurance and self-protection in the case of a specific non-expected utility model-namely, the "rank-dependent" functional form of Quiggin [1982]. They find that most (though not all) of the expected utility-based results on self-insurance generalize to this non-expected utility model, whereas the generally ambiguous results on self-protection ${ }^{31}$ must, of necessity, remain ambiguous in this more general setting. 32

A treatment anywhere near as extensive as Konrad and Skaperdas's analysis is beyond the scope of this paper. However, we do examine what is probably the most basic theorem of self-insurance-namely, that greater risk aversion leads to greater self-insurance, which was proven by Dionne and Eeckhoudt [1985] for expected utility and Konrad and Skaperdas [1993, prop. 1] for the non-expected utility "rank-dependent" functional form. Here we formally show that this comparative statics result extends to all smooth risk averse nonexpected utility maximizers, whether or not they are outcome convex:

Theorem 3. Assume that there are two states of nature with fixed positive probabilities $\bar{p}$ and $(1-\bar{p})$. Let $w_{0}>0$ be base wealth, $\alpha \in[0, M]$ be expenditure on self-insurance, and $\ell(\alpha)>0$ be the loss in the first state, where $\ell^{\prime}(\alpha)<0$ and $M<w_{0}$. Assume that the non-expected utility preference functions ${ }^{\top} \nabla_{1}(\cdot)$ and $\nabla_{2}(\cdot)$ are twice continuously Fréchet 
differentiable, strictly risk averse, and that $\nabla_{1}(\cdot)$ is strictly more risk averse than $\nabla_{2}\left({ }^{\circ}\right)$ in the sense that $-U_{1}^{\prime \prime}(x ; F) / U_{1}^{\prime}(x ; F)>-U_{2}^{\prime \prime}(x ; F) / U_{2}^{\prime}(x ; F)$ for all $x$ and $F(\cdot)$. Consider the problem

$$
\max _{\alpha \in[0, M]} \vartheta_{i}\left(w_{0}-\ell(\alpha)-\alpha, \bar{p} ; w_{0}-\alpha, 1-\bar{p}\right) \quad i=1,2 .
$$

If $\alpha_{1}^{*}$ is the smallest solution to this problem for ${ }^{~} \nabla_{1}(\cdot)$, and $\alpha_{2}^{*}$ is the largest solution for $\nabla_{2}(\cdot)$, then $\alpha_{1}^{*} \geq \alpha_{2}^{*}$, with strict inequality unless $\alpha_{1}^{*}=0$ or $\alpha_{2}^{*}=M$.

\section{Proof. In Appendix.}

In other words, regardless of the possible multiplicity of optima due to non-outcome convexity, we will never observe the more risk-averse first individual choosing less selfinsurance than the second individual, and the only time they would ever choose the same level is if the producitivity of self-insurance is so weak that zero is an optimum even for the first individual (in which case it is the only optimum for the second) or else the productivity is so strong that full self-insurance $(\alpha=M)$ is an optimum even for the second individual (in which case it is the only optimum for the first).

\section{Conclusion}

Although the reader was warned that this robustness check would be more broad than deep, even so, it is of incomplete breadth. There are several important areas of the theory of insurance that remain unexamined. One important area is the effect of changes in risk (as opposed to risk aversion) on the demand for insurance. This has been studied in the expected utility framework by Alarie, Dionne, and Eeckhoudt [1992]. Although any conclusions at this point would be premature, my own work (Machina [1989]) on the robustness of the classic Rothschild-Stiglitz [1971] analysis of the comparative statics of risk suggests that this might be another area in which standard expected utility-based results would generally extend.

Another potentially huge area is that of insurance under asymmetric information. This has already played an important role in the motivation of much of insurance theory, as, for example, in the theory of adverse selection (e.g., Pauly [1974], Rothschild and Stiglitz [1976]) and the theory of moral hazard (e.g., Arrow [1963, 1968], Pauly [1968], Shavell [1979]). Just as this work has been primarily built on the basis of individual expected utility maximization, so, presumably, could it be built on (or at least examined from) the basis of non-expected utility preferences.

A final, perhaps less well-defined area, is that of insurance under situations of ambiguitythat is, the absence of well-defined subjective probabilities. Although formal research on ambiguity and insurance has already begun (e.g., Hogarth and Kunreuther [1989, 1992a, $1992 \mathrm{~b}]$ ), the nature of many non-expected utility models of choice under ambiguity ${ }^{33}$ departs sufficiently from classic expected utility theory that the robustness of standard insurance results to ambiguity is still very much an open question. 
Non-expected utility theory is still in the process of moving from its initial phase of concentrating on "choice paradoxes" and "alternative models" to the subsequent stage of reexamining standard questions in the economics of risk and insurance. As I hope I have shown, we non-expected utility researchers have been, and will continue to be, beholden to the prior work of expected utility theorists in this endeavor. No one should expect a revolution from this new line of research-just some added insight on the relationship between the assumptions of risk and insurance theorems and their conclusions.

\section{Appendix: Proofs of theorems}

\section{Proof of Theorem 1}

For notational simplicity, we can equivalently rewrite (28) as

$$
\max _{\rho \in[0,1]} \nabla_{i}\left(c_{0}+p \cdot \tilde{z}\right) \quad i=1,2
$$

where $c_{0}=w_{0}-\lambda \cdot E[\tilde{\ell}], \rho \equiv(1-\alpha)$, and $\tilde{z} \equiv \lambda \cdot E[\tilde{\ell}]-\tilde{\ell}$ with cumulative distribution function $F_{\tilde{z}}(\cdot)$. Proving the theorem is then equivalent to proving that if $\rho_{1}^{*}$ is the largest solution to (A.1) for $\nabla_{1}\left({ }^{\circ}\right)$ and $\rho_{2}^{*}$ is the smallest solution for $\nabla_{2}\left({ }^{\circ}\right.$, then $\rho_{1}^{*} \leq \rho_{2}^{*}$, with strict inequality unless $\rho_{1}^{*}=1$.

For all $\rho \in[0,1]$ and $c \geq c_{0}$, define the preference functions

$$
\phi_{i}(\rho, c) \equiv \nabla_{i}\left(F_{c+\rho \cdot z}\right) \quad i=1,2,
$$

where $F_{c+\rho z \tilde{z}}(\cdot)$ is the cumulative distribution function of the random variable $c+\rho \cdot \tilde{z}$. By construction, each function $\phi_{i}(\rho, c)$ is continuously differentiable and possesses indifference curves over the set $\left\{(\rho, c) \mid \rho \in[0,1], c \geq c_{0}\right\}$, which are "inherited" from $\nabla_{i}\left({ }^{\circ}\right)$, as in Figure A.1. Since first-order stochastic dominance preference ensures that $\partial \phi_{i}(\rho, c) / \partial c>0$, these indifference curves cannot be either backward bending or forward bending, although they can be either upward or downward sloping or both. Note that the horizontal line $c=c_{0}$ in the figure corresponds to the one-dimensional feasible set in the maximization problem (A.1). In other words, $\phi_{i}\left(\rho, c_{0}\right)$ equals the objective function in (A.1), so $\rho_{1}^{*}$ and $\rho_{2}^{*}$ are the largest and the smallest global maxima of $\phi_{1}\left(\rho, c_{0}\right)$ and $\phi_{2}\left(\rho, c_{0}\right)$, respectively.

We first show that, at any point in the set $\left\{(\rho, c) \mid \rho \in(0,1), c \geq c_{0}\right\}$, the marginal rates of substitution for the preference functions $\phi_{1}(\rho, c)$ and $\phi_{2}(\rho, c)$ must satisfy

$$
M R S_{1}(\rho, c) \equiv-\frac{\partial \phi_{1}(\rho, c) / \partial \rho}{\partial \phi_{1}(\rho, c) / \partial c}>-\frac{\partial \phi_{2}(\rho, c) / \partial \rho}{\partial \phi_{2}(\rho, c) / \partial c} \equiv M R S_{2}(\rho, c)
$$

To demonstrate this inequality, assume it is false, so that at some such point $(\rho, c)$ we had ${ }^{34}$

$$
-\frac{\partial \phi_{1}(\rho, c) / \partial \rho}{\partial \phi_{1}(\rho, c) / \partial c} \leq k \leq-\frac{\partial \phi_{2}(\rho, c) / \partial \rho}{\partial \phi_{2}(\rho, c) / \partial c}
$$

for some value $k$. Since $k$ could have any sign, $c-\rho \cdot k$ could be either negative or nonnegative. 
If $c-\rho \cdot k<0$ : In this case, $c-\rho \cdot \tilde{z} \geq 0^{35}$ implies $\rho \cdot \tilde{z}+\rho \cdot k>0$ and hence $\tilde{z}+k>0$, which implies

$$
0<\int(z+k) \cdot U_{2}^{\prime}\left(c+\rho \cdot z ; F_{c+\rho ; z}\right) \cdot d F_{\tilde{z}}(z)
$$

(A.5), (15), and (A.2) imply

$k>-\frac{\int z \cdot U_{2}^{\prime}\left(c+\rho \cdot z ; F_{c+\rho} \tilde{z}\right) \cdot d F_{\tilde{z}}(z)}{\int U_{2}^{\prime}\left(c+\rho \cdot z ; F_{c+\rho \cdot \tilde{z}}\right) \cdot d F_{\tilde{z}}(z)}=-\frac{\partial \nabla_{2}(c+\rho \cdot \tilde{z}) / \partial \rho}{\partial \nabla_{2}(c+\rho \cdot \tilde{z}) / \partial c}=-\frac{\partial \phi_{2}(\rho, c) / \partial \rho}{\partial \phi_{2}(\rho, c) / \partial c}$

which is a contradiction, since it violates (A.4).

If $c-\rho \cdot k \geq 0:$ In this case, (A.4), (A.2), and (15) imply

$$
k \geq-\frac{\partial \nabla_{1}\left(F_{c+\rho \cdot \tilde{z}}\right) / \partial \rho}{\partial V_{1}\left(F_{c+\rho \cdot \tilde{z}}\right) / \partial c}=-\frac{\int z \cdot U_{1}^{\prime}\left(c+\rho \cdot z ; F_{c+\rho \cdot \tilde{z}}\right) \cdot d F_{\tilde{z}}(z)}{\int U_{1}^{\prime}\left(c+\rho \cdot z ; F_{c+\rho \cdot \tilde{z}}\right) \cdot d F_{\tilde{z}}(z)}
$$

so that we have

$$
\begin{aligned}
& 0 \leq \int(z+k) \cdot \frac{U_{1}^{\prime}\left(c+\rho \cdot z ; F_{c+\rho} \tilde{z}\right)}{U_{1}^{\prime}\left(c-\rho \cdot k ; F_{c+\rho \cdot \tilde{z}}\right)} \cdot d F_{\tilde{z}}(z) \\
& =\int_{z+k>0}(z+k) \cdot \frac{U_{1}^{\prime}\left(c+\rho \cdot z ; F_{c+\rho \cdot \tilde{z}}\right)}{U_{1}^{\prime}\left(c-\rho \cdot k ; F_{c+\rho \cdot \tilde{z}}\right)} \cdot d F_{\tilde{z}}(z) \\
& +\int_{z+k<0}(z+k) \cdot \frac{U_{1}^{\prime}\left(c+\rho \cdot z ; F_{c+\rho \cdot \tilde{z}}\right)}{U_{1}^{\prime}\left(c-\rho \cdot k ; F_{c+\rho \cdot \tilde{z}}\right)} \cdot d F_{\tilde{z}}(z) \\
& <\int_{z+k>0}(z+k) \cdot \frac{U_{2}^{\prime}\left(c+\rho \cdot z ; F_{c+\rho \cdot z}\right)}{U_{2}^{\prime}\left(c-\rho \cdot k ; F_{c+\rho \cdot \tilde{z}}\right)} \cdot d F_{\tilde{z}}(z) \\
& +\int_{z+k<0}(z+k) \cdot \frac{U_{2}^{\prime}\left(c+\rho \cdot z ; F_{c+\rho \cdot \tilde{z}}\right)}{U_{2}^{\prime}\left(c-\rho \cdot k ; F_{c+\rho \cdot \tilde{z}}\right)} \cdot d F_{\tilde{z}}(z) \\
& =\int(z+k) \cdot \frac{U_{2}^{\prime}\left(c+\rho \cdot z ; F_{c+\rho \cdot \tilde{z}}\right)}{U_{2}^{\prime}\left(c-\rho \cdot k ; F_{c+\rho \cdot \tilde{z})}\right.} \cdot d F_{\tilde{z}}(z)
\end{aligned}
$$

where the strict inequality for the " $z+k>0$ " integrals follows since in this case we have $c+\rho \cdot z>c-\rho \cdot k$, so comparative risk aversion implies $0<U_{1}^{\prime}(c+\rho \cdot z$; 
$\left.F_{c+\rho \cdot \tilde{z}}\right) / U_{1}^{\prime}\left(c-\rho \cdot k ; F_{c+\rho \cdot \tilde{z}}\right)<U_{2}^{\prime}\left(c+\rho \cdot z ; F_{c+\rho \cdot \tilde{z}}\right) / U_{2}^{\prime}\left(c-\rho \cdot k ; F_{c+\rho \cdot z)}\right)$. Strict inequality for the " $z+k<0$ " integrals follows since in this case we have $c+\rho \cdot z<c-\rho \cdot k$, so the comparative-risk-aversion condition implies $U_{1}^{\prime}\left(c+\rho \cdot z ; F_{c+\rho \cdot z}\right) / U_{1}^{\prime}(c-\rho \cdot k$; $F_{c+\rho \cdot z)}>U_{2}^{\prime}\left(c+\rho \cdot z ; F_{c+\rho \cdot z)}\right) / U_{2}^{\prime}\left(c-\rho \cdot k ; F_{c+\rho \cdot z)}\right)>0$, but these ratios are each multiplied by the negative quantity $(z+k)$. This once again implies (A.5) and hence (A.6) and a contradiction. This then establishes inequality (A.3).

Inequality (A.3) implies that, throughout the entire region $\left\{(\rho, c) \mid \rho \in(0,1), c \geq c_{0}\right\}$, leftward movements along any $\phi_{1}(\rho, c)$ indifference curve must strictly lower $\phi_{2}(\rho, c)$, and rightward movements along any $\phi_{2}(\rho, c)$ indifference curve must strictly lower $\phi_{1}(\rho, c)$.

Assume $\rho_{2}^{*}<\rho_{1}^{*}$, as illustrated in Figure A.1. In this case, consider the point $\left(\rho_{2}^{*}, c_{0}\right)$. As we move rightward along the $\phi_{2}(\rho, c)$ indifference curve that passes through this point, the value of $\phi_{1}(\rho, c)$ must strictly drop, so that $\phi_{1}(\rho, c)$ strictly prefers the point $\left(\rho_{2}^{*}, c_{0}\right)$ to every point on the curve that lies to the right of $\left(\rho_{2}^{*}, c_{0}\right)$. But since $\left(\rho_{2}^{*}, c_{0}\right)$ is a global optimum for $\phi_{2}\left(\rho, c_{0}\right)$, this indifference curve must lie everywhere on or above the horizontal line $c=c_{0}$. Since $\partial \phi_{1}(\rho, c) / \partial c>0$, this implies that $\phi_{1}(\rho, c)$ strictly prefers the point $\left(\rho_{2}^{*}, c_{0}\right)$ to every point on the line $c=c_{0}$ that lies to the right of $\left(\rho_{2}^{*}, c_{0}\right)$, which contradicts the assumption that there is a global maximum $\rho_{1}^{*}$ that exceeds (lies to the right of) $\rho_{2}^{*}$. This, then, establishes that $\rho_{1}^{*} \leq \rho_{2}^{*}$.

To complete the proof, we must rule out $\rho_{1}^{*}=\rho_{2}^{*}$ unless $\rho_{1}^{*}=1$. In the case $\rho_{1}^{*}<1$, CO. 2 and $\lambda>1$ imply $\alpha_{2}^{*}<1$ so we would have $0<\rho_{2}^{*}=\rho_{1}^{*}<1$. However this case of identical interior optima would imply that both individuals' indifference curves had zero slope at the interior point $\left(\rho_{1}^{*}, c_{0}\right)=\left(\rho_{2}^{*}, c_{0}\right)$, which violates (A.3).

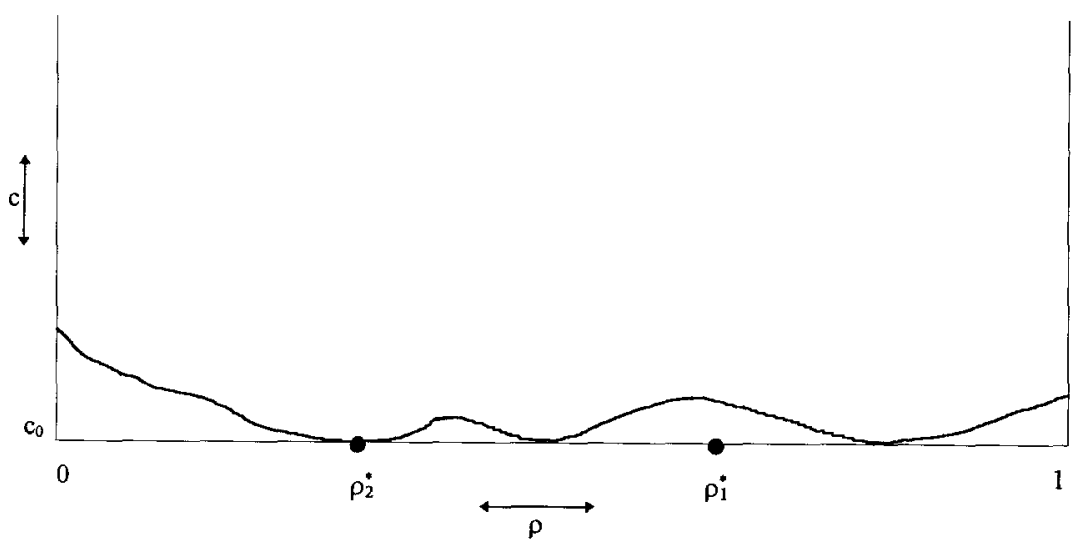

Figure A.1. Indifference curve for the preference function $\phi_{2}(\rho, c)$. 


\section{Proof of Theorem 2}

For notational simplicity, define

$$
\begin{aligned}
\eta(\ell, \alpha) \equiv \lambda \cdot E[\max \{\tilde{\ell}-\alpha, 0\}]+\ell-\max \{\ell-\alpha, 0\}= \\
\left\{\begin{array}{l}
\lambda \cdot \int_{\alpha}^{M}(\epsilon-\alpha) \cdot d F_{\tilde{\ell}}(\epsilon)+\alpha \text { if } \ell \geq \alpha \\
\lambda \cdot \int_{\alpha}^{M}(\epsilon-\alpha) \cdot d F_{\widetilde{\ell}}(\epsilon)+\ell \text { if } \ell<\alpha
\end{array}\right.
\end{aligned}
$$

This implies $\eta(\ell, \alpha)=\eta(\alpha, \alpha)$ if $\ell \geq \alpha$, and $\eta(\ell, \alpha)<\eta(\alpha, \alpha)$ if $\ell>\alpha$. We also have

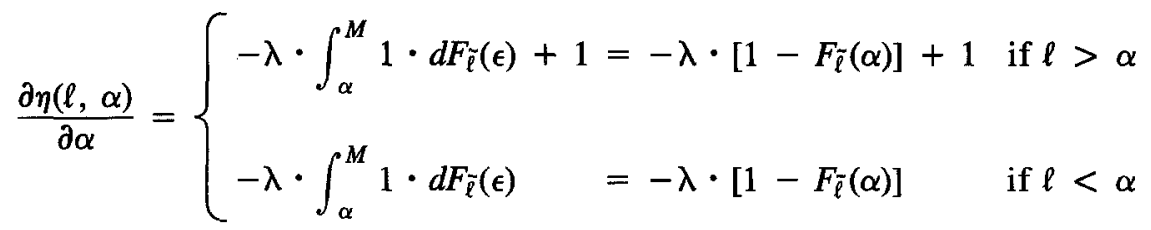

For all $\alpha \in[0, M]$ and $w \geq w_{0}$, let $F_{\alpha, w}(\cdot)$ denote the cumulative distribution function of the random variable

$$
w-\lambda \cdot E[\max \{\tilde{\ell}-\alpha, 0\}]-\tilde{\ell}+\max \{\tilde{\ell}-\alpha, 0\} \equiv w-\eta(\tilde{\ell}, \alpha)
$$

and define the preference functions

$$
\phi_{i}(\alpha, w) \equiv \vartheta_{i}\left(F_{\alpha, w}\right) \quad i=1,2 .
$$

By construction, each function $\phi_{i}(\alpha, w)$ is continuously differentiable and possesses indifference curves over the set $\left\{(\alpha, w) \mid \alpha \in[0, M], w \geq w_{0}\right\}$, which are "inherited" from $\nabla_{i}(\cdot)$, as in Figure A.2. Since first-order stochastic dominance preference ensures that $\partial \phi_{i}(\alpha, w) / \partial w>0$, these indifference curves cannot be either backward bending or forward bending, although they can be either upward or downward sloping or both. Note that the horizontal line $w=w_{0}$ in the figure corresponds to the one-dimensional feasible set in the problem (35). In other words, $\phi_{i}\left(\alpha, w_{0}\right)$ equals the objective function in (35), so $\alpha_{1}^{*}$ and $\alpha_{2}^{*}$ are the largest and the smallest global maxima of $\phi_{1}\left(\alpha, w_{0}\right)$ and $\phi_{2}\left(\alpha, w_{0}\right)$, respectively.

We first show that, at any point in the set $\left\{(\alpha, w) \mid \alpha \in(0, M), w \geq w_{0}\right\}$, the marginal rates of substitution for the preference functions $\phi_{1}(\alpha, w)$ and $\phi_{2}(\alpha, w)$ must satisfy

$$
M R S_{1}(\alpha, w) \equiv-\frac{\partial \phi_{1}(\alpha, w) / \partial \alpha}{\partial \phi_{1}(\alpha, w) / \partial w}>-\frac{\partial \phi_{2}(\alpha, w) / \partial \alpha}{\partial \phi_{2}(\alpha, w) / \partial w} \equiv \operatorname{MRS}_{2}(\alpha, w)
$$


To demonstrate this inequality, assume it is false, so that at some such point $(\alpha, w)$ we had ${ }^{36}$

$$
-\frac{\partial \phi_{1}(\alpha, w) / \partial \alpha}{\partial \phi_{1}(\alpha, w) / \partial w} \leq k \leq-\frac{\partial \phi_{2}(\alpha, w) / \partial \alpha}{\partial \phi_{2}(\alpha, w) / \partial w}
$$

for some $k$. Since $k$ could have any sign, $k+\lambda \cdot\left[1-F_{\tilde{\ell}}(\alpha)\right]$ could be either nonpositive or positive.

If $k+\lambda \cdot\left[1-F_{\tilde{\ell}}(\alpha)\right] \leq 0$ : In this case, note from (A.10) that at the point $(\alpha, w)$, a differential increase in $\alpha$ of $d \alpha$ combined with a differential change in $w$ of $d w=-\lambda$. $\left[1-F_{\tilde{\ell}}(\alpha)\right] \cdot d \alpha$ has zero differential effect on $w-\eta(\ell, \alpha)$ for each $\ell<\alpha$, and a strictly negative differential effect on $w-\eta(\ell, \alpha)$ for each $\ell>\alpha$. Since $\alpha \in(0, M)$ so that $\operatorname{prob}(\tilde{\ell}>\alpha)>0$, this implies a strictly negative differential effect on $\vartheta_{1}\left(F_{\alpha, w}\right)$. Hence, the value of $d w$ necessary to have zero differential effect on $\nabla_{1}\left(F_{\alpha, w}\right)$ must be greater than $-\lambda \cdot\left[1-F_{\tilde{\ell}}(\alpha)\right] \cdot d \alpha$, and hence greater than $k \cdot d \alpha$. This implies that $M R S_{1}(\alpha, w)>k$, which is a contradiction since it violates (A.14).

If $k+\lambda \cdot\left[1-F_{\tilde{\ell}}(\alpha)\right]>0$ : From (A.10), this implies that $k-\partial \eta(\ell, \alpha) / \partial \alpha>0$ for $\ell<\alpha$. (A.14), (A.12), and (15) imply

$k \geq-\frac{\partial \nabla_{1}\left(F_{\alpha, w}\right) / \partial \alpha}{\partial \nabla_{1}\left(F_{\alpha, w}\right) / \partial w}=-\frac{\int\left[-\frac{\partial \eta(\ell, \alpha)}{\partial \alpha}\right] \cdot U_{1}^{\prime}\left(w-\eta(\ell, \alpha) ; F_{\alpha, w}\right) \cdot d F_{\tilde{\ell}}(\ell)}{\int U_{1}^{\prime}\left(w-\eta(\ell, \alpha) ; F_{\alpha, w}\right) \cdot d F_{\tilde{\ell}}(\ell)}$

so that we have

$$
\begin{aligned}
0 \leq & \int\left[k-\frac{\partial \eta(\ell, \alpha)}{\partial \alpha}\right] \cdot \frac{U_{1}^{\prime}\left(w-\eta(\ell, \alpha) ; F_{\alpha, w}\right)}{U_{1}^{\prime}\left(w-\eta(\alpha, \alpha) ; F_{\alpha, w}\right)} \cdot d F_{\tilde{\ell}}(\ell) \\
= & \int_{\ell \geq \alpha}\left[k-\frac{\partial \eta(\ell, \alpha)}{\partial \alpha}\right] \cdot \frac{U_{1}^{\prime}\left(w-\eta(\ell, \alpha) ; F_{\alpha, w}\right)}{U_{1}^{\prime}\left(w-\eta(\alpha, \alpha) ; F_{\alpha, w}\right)} \cdot d F_{\tilde{\ell}}(\ell) \\
& +\int_{\ell<\alpha}\left[k-\frac{\partial \eta(\ell, \alpha)}{\partial \alpha}\right] \cdot \frac{U_{1}^{\prime}\left(w-\eta(\ell, \alpha) ; F_{\alpha, w}\right)}{U_{1}^{\prime}\left(w-\eta(\alpha, \alpha) ; F_{\alpha, w}\right)} \cdot d F_{\tilde{\ell}}(\ell) \\
= & \int_{\ell \geq \alpha}\left[k-\frac{\partial \eta(\ell, \alpha)}{\partial \alpha}\right] \cdot \frac{U_{1}^{\prime}\left(w-\eta(\alpha, \alpha) ; F_{\alpha, w}\right)}{U_{1}^{\prime}\left(w-\eta(\alpha, \alpha) ; F_{\alpha, w}\right)} \cdot d F_{\tilde{\ell}}(\ell) \\
& +\int_{\ell<\alpha}\left[k-\frac{\partial \eta(\ell, \alpha)}{\partial \alpha}\right] \cdot \frac{U_{1}^{\prime}\left(w-\eta(\ell, \alpha) ; F_{\alpha, w}\right)}{U_{1}^{\prime}\left(w-\eta(\alpha, \alpha) ; F_{\alpha, w}\right)} \cdot d F_{\tilde{\ell}}(\ell) \\
< & \int_{\ell \geq \alpha}\left(k-\frac{\partial \eta(\ell, \alpha)}{\partial \alpha}\right] \cdot \frac{U_{2}^{\prime}\left(w-\eta(\alpha, \alpha) ; F_{\alpha, w}\right)}{U_{2}^{\prime}\left(w-\eta(\alpha, \alpha) ; F_{\alpha, w}\right)} \cdot d F_{\tilde{\ell}}(\ell) \\
& +\int_{\ell<\alpha}\left[k-\frac{\partial \eta(\ell, \alpha)}{\partial \alpha}\right] \cdot \frac{U_{2}^{\prime}\left(w-\eta(\ell, \alpha) ; F_{\alpha, w}\right)}{U_{2}^{\prime}\left(w-\eta(\alpha, \alpha) ; F_{\alpha, w}\right)} \cdot d F_{\tilde{\ell}}(\ell)
\end{aligned}
$$




$$
\begin{aligned}
= & \int_{\ell \geq \alpha}\left(k-\frac{\partial \eta(\ell, \alpha)}{\partial \alpha}\right] \cdot \frac{U_{2}^{\prime}\left(w-\eta(\ell, \alpha) ; F_{\alpha, w}\right)}{U_{2}^{\prime}\left(w-\eta(\alpha, \alpha) ; F_{\alpha, w}\right)} \cdot d F_{\tilde{\ell}}(\ell) \\
& +\int_{\ell<\alpha}\left(k-\frac{\partial \eta(\ell, \alpha)}{\partial \alpha}\right] \cdot \frac{U_{2}^{\prime}\left(w-\eta(\ell, \alpha) ; F_{\alpha, w}\right)}{U_{2}^{\prime}\left(w-\eta(\alpha, \alpha) ; F_{\alpha, w}\right)} \cdot d F_{\tilde{\ell}}(\ell) \\
= & \int\left[k-\frac{\partial \eta(\ell, \alpha)}{\partial \alpha}\right] \cdot \frac{U_{2}^{\prime}\left(w-\eta(\ell, \alpha) ; F_{\alpha, w}\right)}{U_{2}^{\prime}\left(w-\eta(\alpha, \alpha) ; F_{\alpha, w}\right)} \cdot d F_{\tilde{\ell}}(\ell) .
\end{aligned}
$$

Note that the $\ell \geq \alpha$ integrals in the fourth and sixth lines of (A.16) are exactly equal. The strict inequality in (A.16) derives from the $\ell<\alpha$ integrals in these two lines, since for these integrals we have (1) $w-\eta(\ell, \alpha)>w-\eta(\alpha, \alpha)$, so the comparative risk-aversion condition implies $U_{2}^{\prime}(w-\eta(\ell, \alpha)) / U_{2}^{\prime}(w-\eta(\alpha, \alpha))>U_{1}^{\prime}(w-\eta(\ell, \alpha)) / U_{1}^{\prime}(w-\eta(\alpha, \alpha))$ $>0$; (2) the term $(k-\partial \eta(\ell, \alpha) / \partial \alpha)$ is positive; and (3) since $\alpha \in(0, M)$, the distribution $F_{\bar{\ell}}(\cdot)$ assigns positive probability to the range $\ell \in[0, \alpha)$.

From (A.16) we have

$$
0<\int\left[k-\frac{\partial \eta(\ell, \alpha)}{\partial \alpha}\right] \cdot U_{2}^{\prime}\left(w-\eta(\ell, \alpha) ; F_{\alpha, w}\right) \cdot d F_{\tilde{\ell}}(\ell)
$$

and hence

$$
\begin{aligned}
k & >\frac{\int \frac{\partial \eta(\ell, \alpha)}{\partial \alpha} \cdot U_{2}^{\prime}\left(w-\eta(\ell, \alpha) ; F_{\alpha, w}\right) \cdot d F_{\tilde{\ell}}(\ell)}{\int U_{2}^{\prime}\left(w-\eta(\ell, \alpha) ; F_{\alpha, w}\right) \cdot d F_{\tilde{\ell}}(\ell)} \\
& =-\frac{\partial \nabla_{2}\left(F_{\alpha, w}\right) / \partial \alpha}{\partial \nabla_{2}\left(F_{\alpha, w}\right) / \partial w}=-\frac{\partial \phi_{2}(\alpha, w) / \partial \alpha}{\partial \phi_{2}(\alpha, w) / \partial w}
\end{aligned}
$$

which is a contradiction since it violates (A.14). This then establishes inequality (A.13).

Inequality (A.13) implies that, throughout the entire region $\{(\alpha, w) \mid \alpha \in(0, M), w \geq$ $\left.w_{0}\right\}$, leftward movements along any $\phi_{1}(\alpha, w)$ indifference curve must strictly lower $\phi_{2}(\alpha$, $w)$, and rightward movements along any $\phi_{1}(\alpha, w)$ indifference curve must strictly lower $\phi_{1}(\alpha, w)$.

Assume $\alpha_{2}^{*}<\alpha_{1}^{*}$, as illustrated in Figure A.2. In this case, consider the point $\left(\alpha_{2}^{*}, w_{0}\right)$. As we move rightward along the $\phi_{2}(\alpha, w)$ indifference curve that passes through this point, the value of $\phi_{1}(\alpha, w)$ must strictly drop, so that $\phi_{1}(\alpha, w)$ strictly prefers the point $\left(\alpha_{2}^{*}, w_{0}\right)$ to every point on the curve that lies to the right of $\left(\alpha_{2}^{*}, w_{0}\right)$. But since $\left(\alpha_{2}^{*}, w_{0}\right)$ is a global optimum for $\phi_{2}\left(\alpha, w_{0}\right)$, this indifference curve must lie everywhere on or above the horizontal line $w=w_{0}$. Since $\partial \phi_{1}(\alpha, w) / \partial w>0$, this implies that $\phi_{1}(\alpha, w)$ strictly prefers the point $\left(\alpha_{2}^{*}, w_{0}\right)$ to every point on the line $w=w_{0}$ that lies to the right of $\left(\alpha_{2}^{*}, w_{0}\right)$, which contradicts the assumption that there is a global maximum $\alpha_{1}^{*}$ that exceeds (lies to the right of) $\alpha_{2}^{*}$. This, then, establishes that $\alpha_{1}^{*} \leq \alpha_{2}^{*}$. 


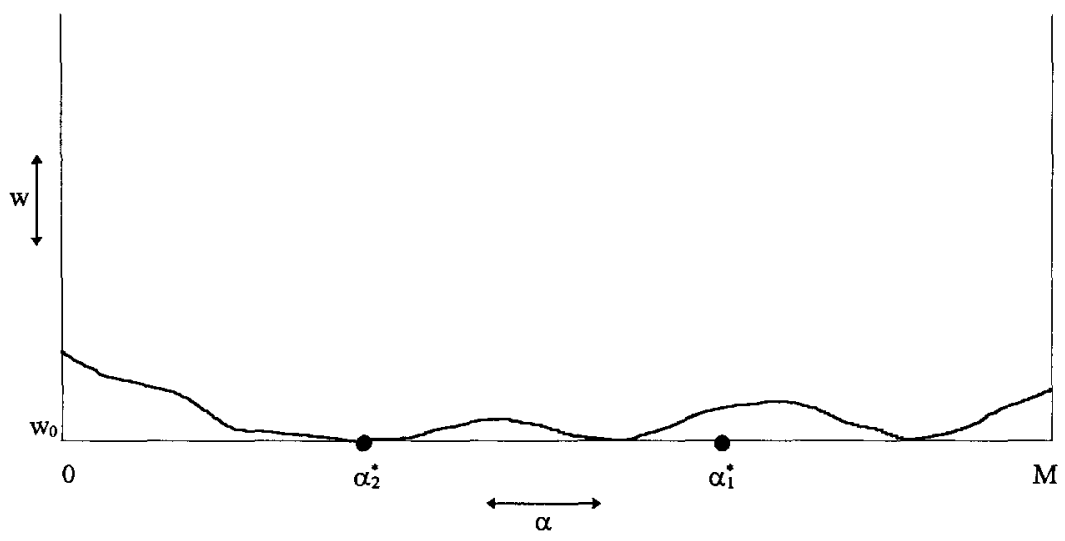

Figure A.2. Indifference curve for the preference function $\phi_{2}(\alpha, w)$.

To complete the proof, we must rule out $\alpha_{1}^{*}=\alpha_{2}^{*}$ unless $\alpha_{1}^{*}=M$. In the case $\alpha_{1}^{*}<M$, DE. 2 and $\lambda>1$ imply $\alpha_{2}^{*}>0$, so that equality of $\alpha_{1}^{*}$ and $\alpha_{2}^{*}$ would imply $0<\alpha_{2}^{*}=$ $\alpha_{1}^{*}<M$. However, this case of identical interior optima would imply that both individuals' indifference curves had zero slope at the interior point $\left(\alpha_{1}^{*}, w_{0}\right)=\left(\alpha_{2}^{*}, w_{0}\right)$, which violates (A.13).

\section{Proof of Theorem 3.}

For all $\alpha \in[0, M]$ and $w \geq w_{0}$, define the probability distribution

$$
P_{\alpha, w} \equiv\left(w_{0}-\ell(\alpha)-\alpha, \bar{p} ; w_{0}-\alpha, 1-\bar{p}\right)
$$

and define the preference functions

$$
\phi_{i}(\alpha, w) \equiv \nabla_{i}\left(P_{\alpha, w}\right) \quad i=1,2 \text {. }
$$

By construction, each function $\phi_{i}(\alpha, w)$ is continuously differentiable and possesses indifference curves over the set $\left\{(\alpha, w) \mid \alpha \in[0, M], w \geq w_{0}\right\}$, which are "inherited" from $\nabla_{i}\left({ }^{\circ}\right)$, as in Figure A.3. Since first-order stochastic dominance preference ensures that $\partial \phi_{i}(\alpha, w) / \partial w>0$, these indifference curves cannot be either backward bending or forward bending, although they can be either upward or downward sloping or both. Note that the horizontal line $w=w_{0}$ in the figure corresponds to the one-dimensional feasible set in the problem (59). In other words, $\phi_{i}\left(\alpha, w_{0}\right)$ equals the objective function in (59), so $\alpha_{1}^{*}$ and $\alpha_{2}^{*}$ are the smallest and largest global maxima of $\phi_{1}\left(\alpha, w_{0}\right)$ and $\phi_{2}\left(\alpha, w_{0}\right)$, respectively.

We first show that, at any point in the set $\left\{(\alpha, w) \mid \alpha \in(0, M), w \geq w_{0}\right\}$, the marginal rates of substitution for the preference functions $\phi_{1}(\alpha, w)$ and $\phi_{2}(\alpha, w)$ must satisfy

$$
M R S_{1}(\alpha, w) \equiv-\frac{\partial \phi_{1}(\alpha, w) / \partial \alpha}{\partial \phi_{1}(\alpha, w) / \partial w}<-\frac{\partial \phi_{2}(\alpha, w) / \partial \alpha}{\partial \phi_{2}(\alpha, w) / \partial w} \equiv M R S_{2}(\alpha, w)
$$


From (A.20) and (15), we have

$$
\begin{aligned}
-\frac{\partial \phi_{1}(\alpha, w) / \partial \alpha}{\partial \phi_{1}(\alpha, w) / \partial w} & =\frac{\left(1+\ell^{\prime}(\alpha)\right) \cdot U_{1}^{\prime}\left(w-\ell(\alpha)-\alpha ; P_{\alpha, w}\right) \cdot \bar{p}+U_{1}^{\prime}\left(w-\alpha ; P_{\alpha, w}\right) \cdot(1-\bar{p})}{U_{1}^{\prime}\left(w-\ell(\alpha)-\alpha ; P_{\alpha, w}\right) \cdot \bar{p}+U_{1}^{\prime}\left(w-\alpha ; P_{\alpha, w}\right) \cdot(1-\bar{p})} \\
& =1+\frac{\ell^{\prime}(\alpha)}{1+\left[\frac{U_{1}^{\prime}\left(w-\alpha ; P_{\alpha, w}\right)}{U_{1}^{\prime}\left(w-\ell(\alpha)-\alpha ; P_{\alpha, w}\right)}\right) \cdot\left[\frac{1-\bar{p}}{\bar{p}}\right]} \\
& <1+\frac{\ell^{\prime}(\alpha)}{1+\left[\frac{U_{2}^{\prime}\left(w-\alpha ; P_{\alpha, w}\right)}{U_{2}^{\prime}\left(w-\ell(\alpha)-\alpha ; P_{\alpha, w}\right)}\right] \cdot\left(\frac{1-\bar{p}}{\bar{p}}\right)} \\
& =-\frac{\partial \phi_{2}(\alpha, w) / \partial \alpha}{\partial \phi_{2}(\alpha, w) / \partial w}
\end{aligned}
$$

where the strict inequality follows since (1) $w-\alpha>w-\ell(\alpha)-\alpha$ so the comparative risk aversion condition implies $U_{2}^{\prime}\left(w-\alpha ; P_{\alpha, w}\right) / U_{2}^{\prime}\left(w-\ell(\alpha)-\alpha ; P_{\alpha, w}\right)>U_{1}^{\prime}(w-\alpha$; $\left.P_{\alpha, w}\right) / U_{1}^{\prime}\left(w-\ell(\alpha)-\alpha ; P_{\alpha, w}\right)>0 ;(2)$ these ratios occur in denominators; and (3) $\ell^{\prime}(\alpha)$ $<0$.

Inequality (A.21) implies that, throughout the entire region $\{(\alpha, w) \mid \alpha \in(0, M), w \geq$ $\left.w_{0}\right\}$, rightward movements along any $\phi_{1}(\alpha, w)$ indifference curve must strictly lower $\phi_{2}(\alpha, w)$, and leftward movements along any $\phi_{2}(\alpha, w)$ indifference curve must strictly lower $\phi_{1}(\alpha, w)$.

Assume $\alpha_{1}^{*}<\alpha_{2}^{*}$, as illustrated in Figure A.3. In this case, consider the point $\left(\alpha_{1}^{*}, w_{0}\right)$. As we move rightward along the $\phi_{1}(\alpha, w)$ indifference curve that passes through this point, the value of $\phi_{2}(\alpha, w)$ must strictly drop, so that $\phi_{2}(\alpha, w)$ strictly prefers the point $\left(\alpha_{1}^{*}, w_{0}\right)$ to every point on the curve that lies to the right of $\left(\alpha_{1}^{*}, w_{0}\right)$. But since $\left(\alpha_{1}^{*}, w_{0}\right)$ is a global

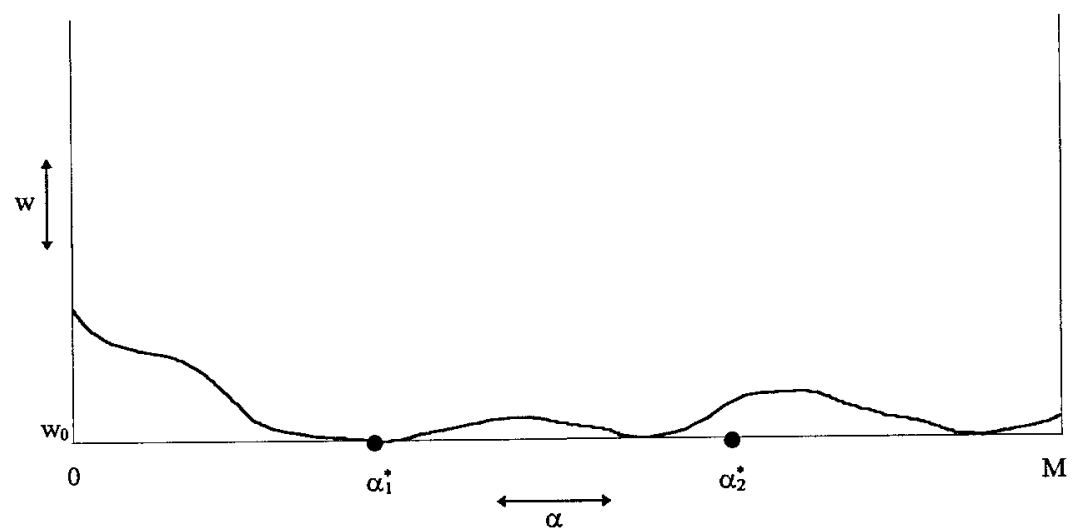

Figure A.3. Indifference curve for the preference function $\phi_{1}(\alpha, w)$. 
optimum for $\phi_{1}\left(\alpha, w_{0}\right)$, this indifference curve must lie everywhere on or above the horizontal line $w=w_{0}$. Since $\partial \phi_{2}(\alpha, w) / \partial w>0$, this implies that $\phi_{2}(\alpha, w)$ strictly prefers the point $\left(\alpha_{1}^{*}, w_{0}\right)$ to every point on the line $w=w_{0}$ that lies to the right of $\left(\alpha_{1}^{*}, w_{0}\right)$, which contradicts the assumption that there is a global maximum $\alpha_{2}^{*}$ that exceeds (lies to the right of) $\alpha_{1}^{*}$. This, then, establishes that $\alpha_{2}^{*} \leq \alpha_{1}^{*}$.

To complete the proof, we must rule out $\alpha_{1}^{*}=\alpha_{2}^{*}$ unless either $\alpha_{1}^{*}=0$ or $\alpha_{2}^{*}=M$. If neither of these cases hold, we have $\alpha_{1}^{*}>0$ and $\alpha_{2}^{*}<M$, so that equality of $\alpha_{1}^{*}$ and $\alpha_{2}^{*}$ would imply $0<\alpha_{1}^{*}=\alpha_{2}^{*}<M$. However, this case of identical interior optima would imply that both individuals' indifference curves had zero slope at the interior point $\left(\alpha_{1}^{*}, w_{0}\right)$ $=\left(\alpha_{2}^{*}, w_{0}\right)$, which violates (A.21).

\section{Acknowledgments}

I would like to thank Christian Gollier for inviting me to present this paper and Michael Carter, Georges Dionne, Christian Gollier, Edi Karni, Mike McCosker, Garey Ramey, Suzanne Scotchmer, Joel Sobel, and Alan Woodfield for helpful comments and suggestions. Support from the National Science Foundation Economics Program and Decision, Risk and Management Science Program (Grant SES 92-09012) is gratefully acknowledged.

\section{Notes}

1. Depending on the context, the probabilities in these distributions can either be actuarially determined chances or a decision maker's personal or subjective probabilities.

2. An interpretive note: The rectangle property is essentially the condition that (smooth) expected utility preferences are separable across mutually exclusive states of nature. Given the rectangle property, the $M R S$ at certainty property is equivalent to state-independent preferences, a property we assume throughout this paper. For important analyses of state-dependent preferences under both expected utility and non-expected utility, see Karni [1985, 1987]. For a specific application to insurance theory, see Cook and Graham [1977].

3. In the standard sense of Rothschild and Stiglitz [1970, 1971].

4. Or, more properly, it will not hold in the general case of $n$ states.

5. As before, they satisfy risk aversion since they are steeper or flatter than the iso-expected value lines in the region above or below the $45^{\circ}$ line, so mean perserving increases in risk make them worse off.

6. For an explicit example, based on the proof of Dekel's Proposition 1 , let $\vee(P) \equiv\left[\Sigma \sqrt{x}_{i} \cdot p_{i}-5\right]^{3}+8$. $\left[\Sigma x_{i} \cdot p_{i}-49\right]^{3}$. Since $[\cdot]^{3}$ is a strictly increasing function over all positive and negative arguments, this preference function is strictly increasing in each $x_{i}$ and satisfies strict first order stochastic dominance preference. Since any mean preserving spread lowers the first bracketed term yet preserves the second, $V(\cdot)$ is also strictly averse. Calculation reveals that $\nabla(\$ 100,1 / 2 ; \$ 0,1 / 2)=\nabla(\$ 49,1 / 2 ; \$ 49,1 / 2)=8$ but $\nabla(\$ 74.5,1 / 2 ; \$ 24.5,1 / 2)$ $\approx 6.74$. But since the latter probability distribution is a $.50: .50$ outcome mixture of the first two, $v\left(\left(^{\circ}\right)\right.$ is not outcome convex.

7. This branch goes by the term generalized expected utility analysis (e.g., Machina [1982, 1983]).

8. Algebraically, $\left\{U\left(x_{i}\right)\right\}$ forms a concave sequence if and only if its point-to-point slopes $\left(U\left(x_{2}\right)-U\left(x_{1}\right)\right)$ / $\left(x_{2}-x_{1}\right),\left(U\left(x_{3}\right)-U\left(x_{2}\right)\right) /\left(x_{3}-x_{2}\right), \ldots$ are successively nonincreasing.

9. $\left\{U_{a}\left(x_{i}\right)\right\}$ is at least as concave than $\left\{U_{b}\left(x_{i}\right)\right\}$ if and only if each ratio of adjacent point-to-point slopes $\left[\left(U\left(x_{i+1}\right)\right.\right.$ $\left.\left.-U\left(x_{i}\right)\right) /\left(x_{i+1}-x_{i}\right)\right] /\left[\left(U\left(x_{i}\right)-U\left(x_{i-1}\right)\right) /\left(x_{i}-x_{i-1}\right)\right]$ is no greater for $\left\{U_{a}\left(x_{i}\right)\right\}$ than for $\left\{U_{b}\left(x_{i}\right)\right\}$.

10. For the appropriate definition of "at least as risk averse as" under non-expected utility, see Machina [1982, 1984].

11. This follows from applying Machina $\left[1982\right.$, eq. 8] to the path $F(* ; \alpha) \equiv\left(x_{1}, p_{1}, \ldots, x_{i-1}, p_{i-1} ; \alpha, p_{i} ; x_{i+1}\right.$, $\left.p_{i+1} ; \ldots ; x_{n}, p_{n}\right)$. 
12. In some of our more formal analysis below (including proofs of theorems), we shall use the natural extension of these ideas to the more general case of a preference functional $V(F)$ over cumulative distribution functions $F(\cdot)$, with local utility function $U(* ; F)$ (see Machina (1982)).

13. The reader wishing an introduction to the vast body of insurance results can do no better than the excellent survey by Dionne and Harrington [1992, pp. 1-48], not to mention the rest of the papers in that volume.

14. This point is nicely made by Karni [1992].

15. Note that this framework abstracts from the problem of uninsurable background risk, as studied, for example, by Doherty and Schlesinger [1983], Schlesinger and Doherty [1985], and Eeckhoudt and Kimball [1992].

16. As demonstrated in Pratt [1964], further results that link increasing or decreasing absolute or relative risk aversion or both to changes in $\alpha$ as an individual's wealth changes can be derived as corollaries of result CO.3.

17. So can result CO.1, if you go and calculate the slope of the budget lines in Figure 7 .

18. This close correspondence of expected utility and non-expected utility first order conditions will come as no surprise to those who have read Chew, Epstein, and Zilcha [1988]. We'll come to this again below.

19. Readers will recognize this argument (and its formalization in the proofs of the theorems) as an application of the well known single-crossing property argument from incentive theory, as in Mirrlees [1971], Spence [1974], and Guesnerie and Laffont [1984], and generalized and extended by Milgrom and Shannon [1994].

20. Thus, $\operatorname{sgn}(\max \{\tilde{\ell}-\alpha, 0\})$ equals 1 when $\ell>\alpha$ and equals 0 when $\ell \leq \alpha$.

21. This was shown by Schlesinger [1981] and Karni [1983].

22. See also the related work in Arrow [1965c, 1974], the subsequent extensions by Raviv [1979] (discussed below), Blazenko [1985], Gollier [1987], and Marshall [1992], and the survey by Gollier [1992, sec. 2].

23. Note, however, that derivative $I^{\prime}(\ell)$ in PE.2 or PE.3 need not be constant, but as Raviv [1979, pp. 90, 91] has shown, depends on both partys' levels of risk aversion as well as marginal indemnity cost $C^{\prime}(I)$.

24. Thus, $\left(I^{*}(\cdot), \pi^{*}\right)$ is a solution to problem (40) for some given $w_{1}$ and $w_{2}$, though it need not be a unique solution.

25. By way of clarification, note that $\Delta \pi$ is a differential change in the scalar $\pi$, while $\Delta I(\cdot)$ is a differential change in the entire function $I(\cdot)$, in the sense being some differential change $\Delta I(\ell)$ in $I(\ell)$ for every value of $\ell$.

26. Readers intrigued by this type of argument are referred to Chew, Epstein, and Zilcha [1988], who, under slightly different assumptions (namely, uniqueness of maxima) demonstrate its astounding generality.

27. See also Gerber [1978], Moffet [1979], Bühlman and Jewell [1979], and Eliashberg and Winkler [1981] for important subsequent contributions, and Lemaire [1990] and Gollier [1992, sec. 1] for insightful surveys.

28. The case of differing beliefs, though clearly more realistic, is beyond the scope of this paper.

29. We say risk tolerance since $\rho_{i}(x)$ is the reciprocal of the standard Arrow-Pratt measure of absolute risk aversion.

30. Like the two-state formula (16), its $n$-state equivalent follows immediately from equation (15).

31. For example, Dionne and Eeckhoudt [1985] show that greater risk aversion can lead to either more or less self-protection.

32. The reader is also referred to Doherty and Eeckhoudt [1994] and Schmidt [1994], which besides Konrad and Skaperdas [1993] and Karni [1992] are the only paper on non-expected utility and insurance of which I am aware. Schmidt studies the non-expected utility functional form of Quiggin [1982] (including the special case of Yaari [1987]), and derives implications for the demand curve for insurance and risk sharing, for these functional forms. Doherty and Eeckhoudt [1994] delve further into the insurance implications of the Yaari [1987] model.

33. See, for example, the survey of Camerer and Weber [1992].

34. From here until the end of the paragraph following (A.8), all equations and discussion refer to this point $(\rho, c)$.

35. Since $c+\rho \cdot \tilde{z} \geq c_{0}+\rho \cdot \tilde{z}=w_{0}-\lambda \cdot E[\tilde{\ell}]+\rho \cdot(\lambda \cdot E[\tilde{\ell}]-\tilde{\ell})=\rho \cdot\left(w_{0}-\tilde{\ell}\right)+(1-\rho) \cdot\left(w_{0}-\right.$ $\lambda \cdot E[\tilde{\ell}])$, nonnegativity of $c+\rho \cdot \tilde{z}$ on the set $\left\{(\rho, c) \mid \rho \in[0,1], c \geq c_{0}\right\}$ follows from nonnegativity of $w_{0}-\tilde{\ell}$ and $w_{0}-\lambda \cdot E[\tilde{\ell}]$. Note that since $c \geq c_{0}>0$, the condition $c-\rho \cdot k<0$ also implies that $\rho$ must be nonzero and hence positive.

36. From here until (A.18), all equations and discussion refer to this point $(\alpha, w)$.

\section{References}

ALARIE, Y., DIONNE, G., and EECKHOUDT, L. [1992]: "Increases in Risk and the Demand for Insurance," in Dionne [1992]. 
ARROW, K. [1963]: "Uncertainty and the Welfare Economics of Medical Care," American Economic Review, 53, 941-969. Reprinted in Arrow [1971] and in part in Diamond and Rothschild [1989].

ARROW, K. [1965a]: Aspects of the Theory of Risk Bearing. Yrjö Jahnsson Säätiö, Helsinki.

ARROW, K. [1965b]: “The Theory of Risk Aversion,” in Arrow [1965a]. Reprinted in Arrow [1971].

ARROW, K. [1965c]: "Insurance, Risk, and Resource Allocation," in Arrow [1965a]. Reprinted in Arrow [1971] and in Dionne and Harrington [1992].

ARROW, K. [1968]: “The Economics of Moral Hazard: Further Comment," American Economic Review, 58, 537-539. Reprinted in Arrow [1971].

ARROW, K. [1971]: Essays in the Theory of Risk-Bearing. North-Holland, Amsterdam.

ARROW, K. [1974]: “Optimal Insurance and Generalized Deductibles," Scandanavian Actuarial Journal, 1, 1-42. BLAZENKO, G. [1985]: “The Design of an Optimal Insurance Policy: Note," American Economic Review, 75, 253-255.

BORCH, K. [1960]: “The Safety Loading of Reinsurance Premiums,” Skandinavisk Aktuarietidskrift, $163-184$. Reprinted in Borch [1990].

BORCH, K. [1961]: “The Utility Concept Applied to the Theory of Insurance," Astin Bulletin, 1, 245-255. Reprinted in Borch [1990].

BORCH, K. [1962]: "Equilibrium in a Reinsurance Market," Econometrica, 30, 424-444. Reprinted in Borch [1990] and in Dionne and Harrington [1992].

BORCH, K. [1990]: Economics of Insurance. North Holland, Amsterdam. (Completed by K. Aase and A. Sandmo.) BOYER, M., and DIONNE, G. [1983]: "Variations in the Probability and Magnitude of Loss: Their Impact on Risk," Canadian Journal of Economics, 16, 411-419.

BOYER, M., and DIONNE, G. [1989]: "More on Insurance, Protection and Risk," Canadian Journal of Economics, 22, 202-205.

BRIYS, E., and SCHLESINGER, H. [1990]: "Risk Aversion and Propensities for Self-Insurance and SelfProtection," Southern Economic Journal, 57, 458-467.

BRIYS, E., SCHLESINGER, H., and SCHULENBERG, J.-M. [1991]: "Reliability of Risk Management: Market Insurance, Self-Insurance, and Self-Protection Reconsidered,' Geneva Papers in Risk and Insurance Theory, 16, 45-58.

BÜHLMAN, E., and JEWELL, H. [1979]: “Optimal Risk Exchanges,” Astin Bulletin, 10, 243-262.

CAMERER, C., and WEBER, M. [1992]: "Recent Developments in Modeling Preferences: Uncertainty and Ambiguity," Journal of Risk and Uncertainty, 5, 325-370.

CHANG, Y.M., and EHRLICH, I. [1985]: “Insurance, Protection from Risk and Risk Bearing," Canadian Journal of Economics, 18, 574-587.

CHEW, S., EPSTEIN, L., and ZILCHA, I. [1988]: “A Correspondence Theorem Between Expected Utility and Smooth Utility," Journal of Economic Theory, 46, 186-193.

COOK, P., and GRAHAM, D. [1977]: "The Demand for Insurance and Protection: The Case of Irreplaceable Commodities," Quarterly Journal of Economics, 91, 143-156. Reprinted in Dionne and Harrington [1992].

DEBREU, G. [1959]: Theory of Value: An Axiomatic Analysis of General Equilibrium. Yale University Press, New Haven.

DEKEL, E. [1989]: “Asset Demands Without the Independence Axiom,” Econometrica, 57, 163-169.

DIAMOND, P., and ROTHSCHILD, M. (eds.) [1989]: Uncertainty in Economics: Readings and Exercises (2nd ed.). Academic Press, New York.

DIONNE, G. (ed.) [1992]: Contributions to Insurance Economics. Kluwer Academic Publishers, Boston (Mass.). DIONNE, G., and EECKHOUDT, L. [1985]: "Self Insurance, Self Protection and Increased Risk Aversion," Economics Letters, 17, 39-42.

DIONNE, G., and HARRINGION, S. (eds.) [1992]: Foundations of Insurance Economics: Readings in Economics and Finance. Kluwer, Boston (Mass.).

DOHERTY, N., and EECKHOUDT, L. [1994]: “Optimal Insurance Without Expected Utility: The Dual Theory and the Linearity of Insurance Contracts," manuscript, University of Pennsylvania.

DOHERTY, N., and SCHLESINGER, H. [1983]: “Optimal Insurance in Incomplete Markets,” Journal of Political Economy, 91, 1045-1054.

DRÈZE, J. [1981]: "Inferring Risk Tolerance from Deductibles in Insurance Contracts," Geneva Papers on Risk and Insurance, 20, 48-52. 
EECKHOUDT, L., GOLLIER, C., and SCHLESINGER, H. [1991]: "Increases in Risk and Deductible Insurance," Journal of Economic Theory, 55, 435-440.

EECKHOUDT, L., and KIMBALL, M. [1992]: "Background Risk, Prudence, and the Demand for Insurance," in Dionne [1992].

EHRLICH, I., and BECKER, G. [1972]: "Market Insurance, Self-Insurance, and Self-Protection," Journal of Political Economy, 80, 623-648. Reprinted in Dionne and Harrington [1992].

ELIASHBERG, J., and WINKLER, R. [1981]: "Risk Sharing and Group Decision Making," Management Science, 27, 1221-1235.

GÄRDENFORS, P., and SAHLIN, N. (eds.) [1988]: Decision, Probability, and Utility: Selected Readings. Cambridge University Press, Cambridge.

GERBER H. [1978]: "Pareto-Optimal Risk Exchanges and Related Decision Problems," Astin Bulletin, 10, 25-33. GOLLIER, C. [1987]: "Pareto-Optimal Risk Sharing with Fixed Costs Per Claim," Scandinavian Actuarial Journal, $13,62-73$.

GOLLIER, C. [1992]: "Economic Theory of Risk Exchanges: A Review," in Dionne [1992].

GOLLIER, C., and SCHLESINGER, H. [1995]: "Arrow's Theorem on the Optimality of Deductibles: A Stochastic Dominance Approach," Economic Theory, forthcoming.

GOULD, J. [1969]: "The Expected Utility Hypothesis and the Selection of Optimal Deductibles for a Given Insurance Policy," Journal of Business, 42, 143-151.

GUESNERIE, R., and LAFFONT, J.-J. [1984]: "A Complete Solution to a Class of Principal-Agent Problems with an Application to the Control of a Self-Managed Firm," Journal of Public Economics, 25, 329-369.

HELPMAN, E., RAZIN, A., and SADKA, E. (eds.) [1983]: Social Policy Evaluation: An Economic Perspective. Academic Press, New York.

HIBERT, L. [1989]: "Optimal Loss Reduction and Risk Aversion," Journal of Risk and Insurance, 56, 300-306.

HIRSHLEIFER, J. [1965]: "Investment Decision Under Uncertainty: Choice-Theoretic Approaches," Quarterly Journal of Economics, 79, 509-536. Reprinted in Hirshleifer [1989].

HIRSHLEIFER, J. [1966]: "Investment Decision Under Uncertainty: Applications of the State-Preference Approach," Quarterly Joumal of Economics, 80, 252-277. Reprinted in Hirshleifer [1989].

HIRSHLEIFER, J. [1989]: Time, Uncertainty, and Information. Basil Blackwell, Oxford.

HIRSHLEIFER, J., and RILEY, J. [1979]: "The Analysis of Uncertainty and Information: An Expository Survey," Journal of Economic Literature, 17, 1375-1421. Reprinted in Hirshleifer [1989].

HIRSHLEIFER, J., and RILEY, J. [1992]: The Analytics of Uncertainty and Information. Cambridge University Press, Cambridge.

HOGARTH, R., and KUNREUTHER, H. [1989]: "Risk, Ambiguity and Insurance," Journal of Risk and Uncertainty, 2, 5-35.

HOGARTH, R., and KUNREUTHER, H. [1992a]: "How Does Ambiguity Affect Insurance Decisions?" in Dionne [1992].

HOGARTH, R. , and KUNREUTHER, H. [1992b]: "Pricing Insurance and Warranties: Ambiguities and Correlated Risks," Geneva Papers on Risk and Insurance Theory, 17, 35-60.

HOY, M., and ROBSON, R. [1981]: "Insurance as a Giffen Good," Economics Letters, 8, 47-51.

KARNI, E. [1983]: "Risk Aversion in the Theory of Health Insurance," in Helpman, Razin, and Sadka [1983].

KARNI, E. [1985]: Decision Making Under Uncertainty: The Case of State Dependent Preferences. Harvard University Press, Cambridge (Mass.).

KARNI, E. [1987]: "Generalized Expected Utility Analysis of Risk Aversion with State-Dependent Preferences," International Economic Review, 28, 229-240.

KARNI, E. [1989]: "Generalized Expected Utility Analysis of Multivariate Risk Aversion," International Economic Review, 30, 297-305.

KARNI, E. [1992]: "Optimal Insurance: A Nonexpected Utility Analysis," in Dionne [1992].

KONRAD, K., and SKAPERDAS, S. [1993]: "Self-Insurance and Self-Protection: A Nonexpected Utility Analysis," Geneva Papers on Risk and Insurance Theory, 18, 131-146.

LEMAIRE, J. [1990]: "Borch's Theorem: A Historical Survey of Applications," in Loubergé [1990].

LOUBERGÉ, H. (ed.) [1990]: Risk, Information and Insurance. Kluwer, Boston (Mass.).

MACHINA, M. [1982]: “ 'Expected Utility’ Analysis Without the Independence Axiom,” Econometrica, 50, 277-323. 
MACHINA, M. [1983]: "Generalized Expected Utility Analysis and the Nature of Observed Violations of the Independence Axiom," in Stigum and Wenstop [1983]. Reprinted in Gärdenfors and Sahlin [1988] and in Dionne and Harrington [1992].

MACHINA, M. [1984]: "Temporal Risk and the Nature of Induced Preferences," Journal of Economic Theory, 33, 199-231.

MACHINA, M. [1989]: "Comparative Statics and Non-Expected Utility Preferences," Joumal of Economic Theory, 47, 393-405.

MARSHALL, J. [1992]: “Optimum Insurance with Deviant Beliefs," in Dionne [1992].

MILGROM, P., and SHANNON, C. [1994]: "Monotone Comparative Statics," Econometrica, 62, $157-180$. MIRRLEES, J. [1971]: “An Exploration in the Theory of Optimal Income Taxation," Review of Economic Studies, 38, 175-208.

MOFFET, D. [1977]: “Optimal Deductible and Consumption Theory,” Journal of Risk and Insurance, 44, 669-682. MOFFET, D. [1979]: “The Risk Sharing Problem," Geneva Papers on Risk and Insurance Theory, 11, 5-13. MOSSIN, J. [1968]: “Aspects of Rational Insurance Purchasing," Journal of Political Economy, 79, 553-568. Reprinted in Dionne and Harrington [1992].

PASHIGIAN, B., , SCHKADE, L., and MENEFEE, G. [1966]: "The Selection of an Optimal Deductible for a Given Insurance Policy," Journal of Business, 39, 35-44.

PAULY, M. [1968]: “The Economics of Moral Hazard," American Economic Review, 58, 531-537.

PAULY, M. [1974]: "Overinsurance and Public Provision of Insurance: The Role of Moral Hazard and Adverse Selection," Quarterly Journal of Economics, 88, 44-62. Reprinted in part in Diamond and Rothschild [1989].

PRATT, J. [1964]: "Risk Aversion in the Small and in the Large," Econometrica, 32, 122-136. Reprinted in Diamond and Rothschild [1989] and in Dionne and Harrington [1992].

QUIGGIN, J. [1982]: “A Theory of Anticipated Utility," Journal of Economic Behavior and Organization, 3, 323-343

RAVIV, A. [1979]: "The Design of an Optimal Insurance Policy," American Economic Review, 69, 84-96. Reprinted in Dionne and Harrington [1992].

ROTHSCHILD, M., and STIGLITZ, J. [1970]: “Increasing Risk: I. A Definition,” Journal of Economic Theory, 2, 225-243. Reprinted in Diamond and Rothschild [1989] and in Dionne and Harrington [1992].

ROTHSCHILD, M., and STIGLITZ, J. [1971]: “Increasing Risk: II. Its Economic Consequences," Journal of Economic Theory, 3, 66-84.

ROTHSCHILD, M., and STIGLITZ, J. [1976]: "Equilibrium in Competitive Insurance Markets: The Economics of Markets with Imperfect Information," Quarterly Journal of Economics, 90, 629-650. Reprinted in Diamond and Rothschild [1989] and in Dionne and Harrington [1992].

SAMUELSON, P. [1960]: “The St. Petersburg Paradox as a Divergent Double Limit," International Economic Review, 1, 31-37.

SCHLESINGER, H. [1981]: “The Optimal Level of Deductibility in Insurance Contracts," Journal of Risk and Insurance, 48, 465-481.

SCHLESINGER, H., and DOHERTY, N. [1985]: "Incomplete Markets for Insurance: An Overview," Journal of Risk and Insurance, 52, 402-423. Reprinted in Dionne and Harrington [1992].

SCHMIDT, U. [1994]: "Demand for Coinsurance and Bilateral Risk-Sharing with Rank-Dependent Utility," manuscript, Institut für Finanzwissenschaft und Sozialpolitik, Kiel.

SHAVELL, S. [1979]: “On Moral Hazard and Insurance," Quarterly Journal of Economics, 93, 541-562. Reprinted in Dionne and Harrington [1992].

SMITH, V. [1968]: “Optimal Insurance Coverage,” Journal of Political Economy, 76, 68-77.

SPENCE, M. [1974]: "Competitive and Optimal Responses to Signals: An Analysis of Efficiency and Distribution," Journal of Economic Theory, 7, 296-332.

STIGUM, B., and WENSTФP, F. (eds.) [1983]: Foundations of Utility and Risk Theory with Applications. D. Reidel Publishing Co., Dordrecht, Holland.

SWEENEY, G., and BEARD, T. [1992]: "The Comparative Statics of Self-Protection," Journal of Risk and Insurance, 59, 301-309.

TOBIN, J. [1958]: "Liquidity Preference as Behavior Toward Risk," Review of Economic Studies, 25, 64-86. WILSON, R. [1968]: "The Theory of Syndicates,” Econometrica, 36, 119-132. 
YAARI, M. [1965]: “Convexity in the Theory of Choice Under Risk," Quarterly Journal of Economics, 79, 278-290.

YAARI, M. [1969]: "Some Remarks on Measures of Risk Aversion and on Their Uses," Journal of Economic Theory, 1, 315-329. Reprinted in Diamond and Rothschild [1989].

YAARI, M. [1987]: "The Dual Theory of Choice Under Risk," Econometrica, 55, 95-115. 